\title{
One central oscillatory drive is compatible with experimental motor unit behaviour in essential and Parkinsonian tremor
}

\author{
Jakob L Dideriksen ，Juan A Gallego , Ales Holobar , Eduardo Rocon ， \\ Jose L Pons and Dario Farina
}

\begin{abstract}
Objective. Pathological tremors are symptomatic to several neurological disorders that are difficult to differentiate and the way by which central oscillatory networks entrain tremorogenic contractions is unknown. We considered the alternative hypotheses that tremor arises from one oscillator (at the tremor frequency) or, as suggested by recent findings from the superimposition of two separate inputs (at the tremor frequency and twice that frequency). Approach. Assuming one central oscillatory network we estimated analytically the relative amplitude of the harmonics of the tremor frequency in the motor neuron output for different temporal behaviors of the oscillator. Next, we analyzed the bias in the relative harmonics amplitude introduced by superimposing oscillations at twice the tremor frequency. These findings were validated using experimental measurements of wrist angular velocity and surface electromyography (EMG) from 22 patients (11 essential tremor, 11 Parkinson's disease). The ensemble motor unit action potential trains identified from the EMG represented the neural drive to the muscles. Main results. The analytical results showed that the relative power of the tremor harmonics in the analytical models of the neural drive was determined by the variability and duration of the tremor bursts and the presence of the second oscillator biased this power towards higher values. The experimental findings accurately matched the analytical model assuming one oscillator, indicating a negligible functional role of secondary oscillatory inputs. Furthermore, a significant difference in the relative power of harmonics in the neural drive was found across the patient groups, suggesting a diagnostic value of this measure (classification accuracy: $86 \%$ ). This diagnostic power decreased substantially when estimated from limb acceleration or the EMG. Signficance. The results indicate that the neural drive in pathological tremor is compatible with one central network providing neural oscillations at the tremor frequency. Moreover, the regularity of this neural oscillation varies across tremor pathologies, making the relative amplitude of tremor harmonics a potential biomarker for diagnostic use.
\end{abstract}

Keywords: pathological tremor, neural drive, motor unit 


\section{Introduction}

Pathological tremor is an involuntary movement of limbs. Essential tremor (ET) and Parkinson's disease (PD) are the two most prevalent neurological disorders causing tremor [1]. Although both disorders have well defined inclusion and exclusion criteria [2], several similar symptoms make the diagnosis challenging, in particular in the early stages of the diseases [3]. Therefore, misdiagnosis is common (30-50\% of patients [4]) and the risk of suboptimal treatment is relatively high [5]. For this reason, potential biomarkers not requiring invasive measures that enable differentiation between ET and PD patients have been investigated extensively. While some of these investigations rely on electromyographic (EMG) signals $[6,7]$, most studies have focused on the statistical properties of the motor output recorded by accelerometers [811] or spirography $[3,12]$.

Among the most promising of these approaches is the observation that the mean power of all tremor harmonics in the power spectrum of the measured acceleration of the affected limb was greater in PD than ET patients [11]. The neurophysiological explanation of the findings, however, was not provided. Although mechanical limb properties can actively affect the spectral properties of the motor output in some cases [13], there is a close link between the neural drive to the muscle and the resulting movement [14]. Therefore, spectral characteristics of tremulous movements presumably reflect specific neural mechanisms for muscle control, although the central neural networks from which tremor in ET and PD originates are currently not fully understood $[15,16]$. Recently, it has been suggested that two separate neural oscillators projecting to the muscle via different pathways are responsible for the spectral peaks at the base tremor frequency and at twice that frequency, respectively $[17,18]$. This may provide a possible explanation for the observed differences across ET and PD patients as it implies that the amplitude of spectral peaks at integer multiples of the tremor frequency reflects the relative strength of two central oscillatory networks rather than the harmonics of one input at the base frequency. However, the explanatory power of the explanation based on a single oscillator for which the regularity determine its harmonics has never been evaluated. In this study, we investigated and compared these two options; namely that the neural drive to muscle (i.e., the discharge timings from the innervating motor neuron pool) in ET and PD patients reflects the projection of either one or two central oscillator. In addition, we further investigated if the properties of the neural drive to muscle explain the potential use of the relative amplitude of the tremor harmonics as new biomarkers for tremor diagnosis.

To address these questions, in this study we first provide theoretical derivations that describe the association between the relative power of the tremor harmonics in the neural drive to muscles and the temporal characteristics (regularity) of the oscillatory drive. Importantly, by expressing the power of the higher harmonics as normalized to the power of the first (tremor frequency), the outcome measure is independent of the tremor amplitude and thus of the tremor severity that varies across patients. The theoretical analysis will provide the fundamental framework for understanding how the temporal characteristics of one single oscillator at the tremor frequency projecting to the motor neurons determine the relative power of the second tremor harmonic (twice the tremor frequency, termed $H 2$ ) with respect to the first tremor harmonic (the tremor frequency, termed $H 1$ ). Furthermore, numerical simulations will show how the superimposition of a secondary oscillator (at $H 2$ ) changes this relative power depending on the relative amplitude and phase between the two oscillators. The analytical results were compared to recordings of wrist angular velocity and high-density surface EMG from the wrist muscles exhibiting tremor in 22 patients with ET or PD, as well as to the neural drive to the muscles, estimated from discharge patterns of multiple motor units from each muscle [19].

\section{Methods}

\section{Analytical derivations}

First, we define the ratio $H 2 / H 1$ between the power of the second and first harmonics oscillatory component of the neural drive to muscles in tremor:

$$
\frac{H 2}{H 1}=\frac{S\left(2 F_{T}\right)}{S\left(F_{T}\right)},
$$

where $H 2$ is the power at the second harmonic (twice the tremor fundamental frequency, $F_{T}$ ),$H 1$ is the power at the tremor fundamental frequency (the first harmonic), and $S$ denotes the power spectrum of the neural drive to the muscle. The ratio $H 2 / H 1$ indicates the decrease in power of the second with respect to the first tremor harmonics: the larger the power decrease, the smaller the ratio $H 2 / H 1$.

In a first approximation, the neural drive to muscles in tremor can be considered as a periodic single train of motor unit discharges, with constant inter-spike interval, equal to the inverse of the tremor frequency:

$$
a_{p}(t)=\sum_{i=-\infty}^{\infty} \delta\left(t-i \frac{1}{F_{T}}\right),
$$

where $a_{p}$ is the train of $i$ periodic discharge times, $t$ denotes time, $\delta$ is a delta-function, and $F_{t}$ is the tremor frequency. The power spectrum $A_{p}$ of equation (2) signal is:

$$
A_{p}(f)=F_{T}^{2} \sum_{n=-\infty}^{\infty} \delta\left(f-n F_{T}\right),
$$

where $f$ denotes the Fourier frequency and $n$ denotes the harmonics number. For a pure periodic train of discharges, as in equations (2) and (3), the ratio $H 2 / H 1$ is equal to 1 because all tremor harmonics have the same power. The description of the neural drive in equation (2) corresponds to an exactly periodic signal, without any variability over time. As we will show in the following, deviations from this ideal condition that include variability in the neural drive over time (pseudoperiodic (pp) condition) have the effect of reducing the ratio 
$H 2 / H 1$, so that this ratio is maximum and equal to 1 for perfect regularity (periodic signal) and decreases for increasing degree of irregularity.

To associate the ratio $H 2 / H 1$ to the regularity of the neural signal, we now consider a small variation of the model of neural drive described in equation (2) where random variability in the timing of each tremor oscillation is introduced, yielding a pp spike train:

$$
a_{\mathrm{pp}}(t)=\sum_{i=-\infty}^{\infty} \delta\left(t-i \frac{1}{F_{T}}+q_{i}\right)
$$

where $q_{i}$ are independent, identically distributed random variables that describe the irregularity of inter-pulse intervals. In this case, the power spectrum of $a_{\mathrm{pp}}$ is the sum of a line spectrum and a continuous spectrum [20]:

$$
\begin{aligned}
A_{\mathrm{pp}}(f)= & \frac{1}{F_{T}}\left(1-|Q(f)|^{2}\right)+\frac{1}{F_{T}{ }^{2}}|Q(f)|^{2} \\
& \times \sum_{n=-\infty}^{\infty} \delta\left(f-n F_{T}\right),
\end{aligned}
$$

where $Q$ is the Fourier transform of the probability density function of $q$ (and thus the inter-spike interval histogram). The continuous spectrum (defined by the first term in equation (5)) has values for a certain interval of frequencies, while the line spectrum (last term in equation (5)) has values at discrete frequencies. The amplitudes of both depend on the tremor burst variability. In this way, a near-regular spike train (narrow distribution of $q$ ) has a broader frequency support (and thus a greater ratio $H 2 / H 1$ ) compared to a highly variable one (wide distribution of $q$ ) (figures 1(A) and (B). The more irregular is the train, the smaller the ratio $H 2 / H 1$ is. applied. In this case, assuming that the interval between action potentials does not change across bursts, the neural drive is represented by a periodic (case of equation (2)) or a pp (equation (4)) signal with the burst defined by the function $\varphi$, as follows:

$$
\varphi(t)=\left\{\begin{array}{c}
\delta\left(t-\frac{d}{2}\right)+\delta\left(t+\frac{d}{2}\right) \text { for doublets, } \\
\delta(t-d)+\delta(t)+\delta(t+d) \text { for triplets }
\end{array}\right.
$$

where $d$ is the inter-spike interval between the pulses of the doublet/triplet.

The power spectrum of $\varphi$ is obtained as:

$$
\phi(f)=\left\{\begin{array}{c}
\left|\mathrm{e}^{2 \pi \mathrm{i} \frac{d}{2} f}+\mathrm{e}^{-2 \pi \mathrm{i} \frac{d}{2} f}\right|^{2}=\left|2 \cos \left(2 \pi \frac{d}{2} f\right)\right|^{2} \\
=|2 \cos (\pi d f)|^{2} \text { for doublets } \\
\left|\mathrm{e}^{2 \pi \mathrm{i} d f}+1+\mathrm{e}^{-2 \pi \mathrm{i} d f}\right|^{2}=|1+2 \cos (2 \pi d f)|^{2} \\
=|1+2 \cos (2 \pi d f)|^{2} \text { for triplets }
\end{array} .\right.
$$

For the pure periodic case (equations (2) and (3)), the spectrum of the neural drive to the muscle is:

$$
B_{p}(f)=\left\{\begin{array}{c}
|2 \cos (\pi d f)|^{2} F_{T}^{2} \sum_{n=-\infty}^{\infty} \delta \\
\times\left(f-n F_{T}\right) \text { for doublets } \\
|1+2 \cos (2 \pi d f)|^{2} F_{T}^{2} \sum_{n=-\infty}^{\infty} \delta \\
\times\left(f-n F_{T}\right) \text { for triplets }
\end{array} .\right.
$$

and for the pp it is (equations (4) and (5)):

$$
B_{p p}(f)=\left\{\begin{array}{l}
|2 \cos (\pi d f)|^{2}\left(\frac{1}{F_{T}}\left(1-|Q(f)|^{2}\right)+\frac{1}{F_{T}^{2}}|Q(f)|^{2} \sum_{n=-\infty}^{\infty} \delta\left(f-n F_{T}\right)\right) \text { for doublets } \\
|1+2 \cos (2 \pi d f)|^{2}\left(\frac{1}{F_{T}}\left(1-|Q(f)|^{2}\right)+\frac{1}{F_{T}^{2}}|Q(f)|^{2} \sum_{n=-\infty}^{\infty} \delta\left(f-n F_{T}\right)\right) \text { for triplets }
\end{array} .\right.
$$

Next, we consider the more realistic case of tremorogenic oscillations of the neural drive determined by trains of action potentials that present multiple activations for each tremor burst, as is often observed in experimental conditions $[21,22]$. Here we consider the cases of two and three discharges per burst, henceforth termed doublets and triplets, respectively. It should be noted that this definition of the term 'doublet' is broader than that used elsewhere (e.g. [23, 24]), as no specific upper limit of the inter-spike interval was
Finally, we consider the occurrence of a large number of action potentials per tremor bursts, reflecting the situation in which several motor units exhibit the oscillatory behavior. In this case, the ensemble of activation timings may be approximated either as a square wave (uniform neural activation in the tremor burst) or as a sinusoid (greater activation in the center of the burst) (figures $1(\mathrm{C})$ and (E)).

The power spectrum of the neural drive with square approximation is the following:

$$
C_{\text {square }}(f)=\sin c(f \cdot b w)^{2}\left(\frac{1}{F_{T}}\left(1-|Q(f)|^{2}\right)+\frac{1}{F_{T}^{2}}|Q(f)|^{2} \sum_{n=-\infty}^{\infty} \delta\left(f-n F_{T}\right)\right)
$$


A

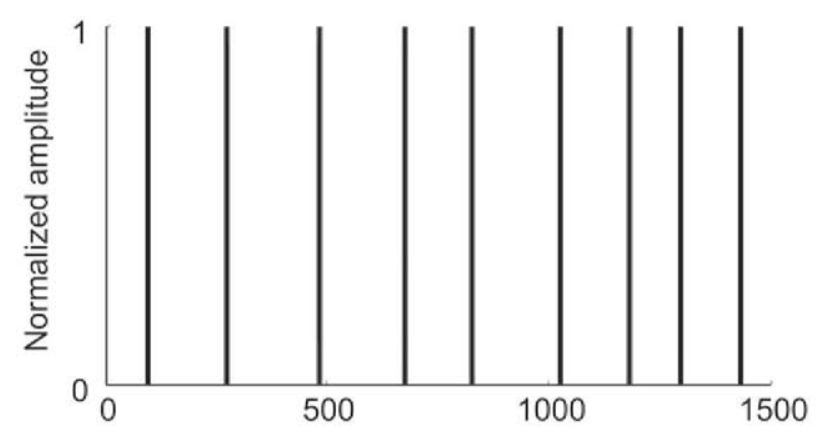

C

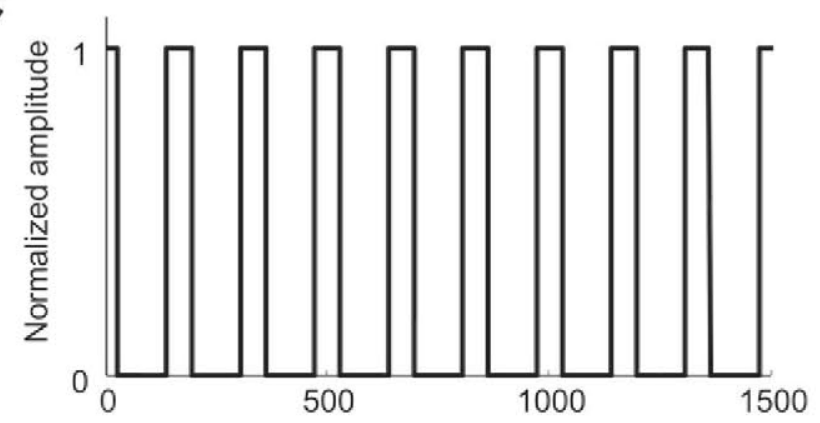

E

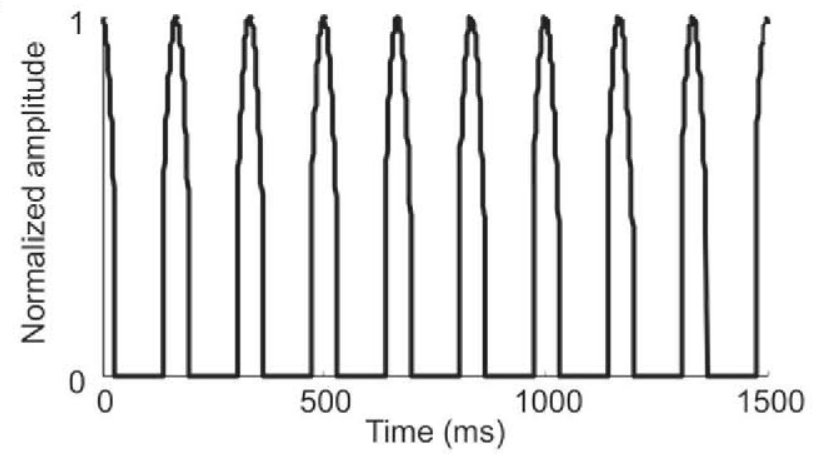

B

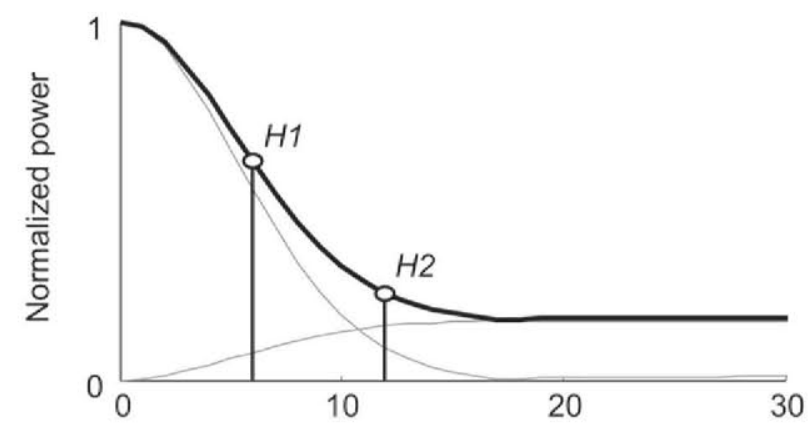

D

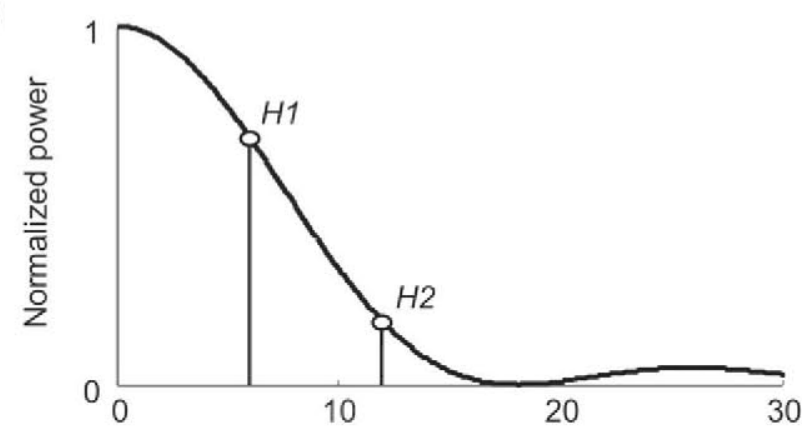

F

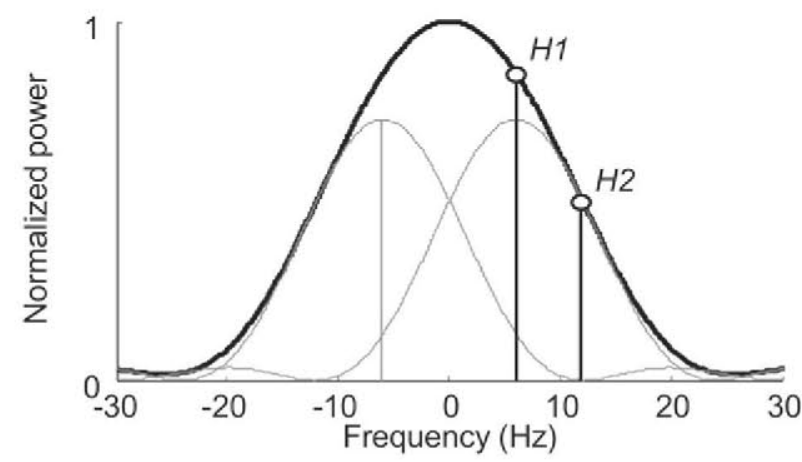

Figure 1. Time- and frequency-domain representations of the tremor-related neural signals. A spike train at $6 \mathrm{~Hz}$ with a coefficient of variation of $18 \%$ (A) has a power spectrum defined by the summation of a line spectrum and a continuous spectrum (gray lines in B). This implies a $H 2 / H 1$ of 0.38 . A square pulse with a width of $55 \mathrm{~ms}$ (33\% of the tremor period) (C) has a power spectrum depicted in (D) and a $H 2 / H 1$ of 0.26 . The same square pulse multiplied by a sinusoid at the tremor frequency (E) has a power spectrum defined as the convolution of Delta functions in \pm tremor frequency and the $\sin c$ function describing the power spectrum of the square wave (gray lines) (F). The $H 2 / H 1$ is 0.58 .

where $b w$ indicates the tremor burst duration. With sinusoid (limited to window with interval $b w$ centered around each peak) approximation, we obtain: and we can thus predict theoretically the influence of the temporal properties of the tremorogenic neural drive on this ratio. Despite the approximations made seem substantial, it

$$
C_{\sin u s}(f)=\left(\sin c(f \cdot b w)^{2} * \frac{1}{4}\left[\delta\left(f+F_{T}\right)+\delta\left(f-F_{T}\right)\right]\right) \times\left(\frac{1}{F_{T}}\left(1-|Q(f)|^{2}\right)+\frac{1}{F_{T}^{2}}|Q(f)|^{2} \sum_{n=-\infty}^{\infty} \delta\left(f-n F_{T}\right)\right)
$$

By substitution of equations (8) and (9) or (10) and (11) in equation (1), we can obtain analytically the ratio $H 2 / H 1$ will be shown that these theoretical predictions can be fully validated from experimental data and therefore hold with a 
good accuracy. The main information provided by the theoretical analysis is that the ratio $H 2 / H 1$ reaches the maximum value of 1 only when the neural activation to the muscle is exactly periodical, without variability. Any source of variability would decrease this ratio, as it will be shown in the Results.

Finally, we observe that the commonly used interference surface EMG and mechanical tremor signals (e.g., force, joint rotation, or acceleration) are obtained by filtering the neural drive to muscles with the single unit action potentials (EMG) or with the mechanical response of the system (mechanical signals):

$$
d(t)=\left\{\begin{array}{l}
\sum_{i=1}^{N} X_{i}(t)^{*} g_{i}(t) \text { for EMG, } \\
\sum_{i=1}^{N} X_{i}(t)^{*} h_{i}(t) \text { for force, }
\end{array}\right.
$$

where $N$ is the number of active motor units, $X$ is the spike train of the $i$ th unit and $g$ and $h$ represent the shape of the motor unit action potential and the motor unit twitch force, respectively. Equation (12) can be simplified by assuming that all motor units generate the same (average) motor unit action potential $(\bar{g})$ and the same (average) twitch force $(\bar{h})$, respectively. Under this approximation, the power spectrum of the electrophysiological or mechanical signal $\left(D_{t}\right)$ representing tremor is:

$$
D(f)=\left\{\begin{array}{l}
C(f)|\mathscr{F}(\bar{g}(t))|^{2} \text { for EMG, } \\
C(f)|\mathscr{F}(\bar{h}(t))|^{2} \text { for force },
\end{array}\right.
$$

where $C$ represents the power spectrum of the neural drive as in equations (10) and (11) and $\mathscr{F}$ is the Fourier transform operator. Equation (13) indicates that the EMG or force (equivalent for acceleration) signals are filtered versions of the neural drive to muscles. The effect of the filters on the relative power of tremor harmonics $(H 2 / H 1)$ is obtained by substituting equation (13) in equation (1). The Results will show that, because the average action potential and twitch waveforms vary across subjects, muscles, and conditions, their filtering effect partly hinders the discriminatory power of the neural drive to muscle for identifying the tremor pathology.

\section{Simulations}

To simulate the impact of an additional neural input at twice the tremor frequency on the ratio $H 2 / H 1$, the tremorogenic pulses in the neural drive was approximated as square pulses. A series of square pulses at the $5 \mathrm{~Hz}$ and a burst width of $30 \%$ of the period was defined and the $H 2 / H 1$ was estimated from its power spectrum as a reference value. Next, another series of square pulses at twice the tremor frequency was superimposed and the change (gain) in the $H 2 / H 1$ with respect to the reference value was estimated. The simulations varied the relative amplitude of the two signals (amplitude of the superimposed square pulses were $0,12.5,25,37.5$, and $50 \%$ of the amplitude of the pulses at the tremor frequency) as well as their relative phase ( $0-2 \pi$ in 10 steps). In addition, the simulations were carried out with three different levels of variability of the pulses. By imposing independent random, Gaussian noise on the timing of the burst centers, the coefficient of variation $(\mathrm{CoV})$ of the inter-burst interval of each of the two series of pulses was 0,5 , or $10 \%$. Each condition was repeated 50 times and the average $H 2 / H 1$ was obtained.

\section{Experimental setup}

We recorded data from $11 \mathrm{ET}$ patients ( 4 female, 7 male; age, mean \pm SD: $70.5 \pm 4.3$ years, range $65-79$ years) and $11 \mathrm{PD}$ patients ( 4 female, 7 male; age, mean \pm SD: $69.0 \pm 6.8$ years, range $60-80$ years). Patients were recruited by neurologists at Hospital Universitario '12 de Octubre', Madrid, Spain. Before the experiments, they signed a written informed consent to participate. The study was performed in accordance with the Declaration of Helsinki and the ethical committee of Hospital Universitario ' 12 de Octubre' approved the experimental protocol. ET patients had a diagnosis of definite ET according to the criteria of the Tremor Investigation Group and the consensus of the Movement Disorder Society [2], and PD patients met the UK PD Society brain Bank Clinical Diagnostic Criteria [25]. No patient had a history of neurological disease other than that causing the tremor. Tremor severity ranged from mild to severe (Fahn-Tolosa-Marin score [26]: $33.7 \pm 12.2$ (range 15-51) for ET patients and UPDRS score [27]: $18.9 \pm 13.0$ (range 5-51) for PD patients). Eight ET patients were taking anti-tremor drugs (mysoline, two patients; propranolol, two patients; clonazepam, one patient; gabapentine, one patient; mysoline and propanolol, one patient; zonisamide, alprazolam and mysoline, one patient), which were continued during the recordings. Nine PD patients received medication at the time of the recordings (levodopa, two patients; levodopa and rasagiline, one patient; levodopa and rotigotine, one patient; levodopa and ropinirole, one patient; rasagiline, one patient; rasagiline and pramipexole, three patients).

Surface EMG was recorded using four $13 \times 5$ electrode grids with an inter-electrode distance (IED) of $8 \mathrm{~mm}$ (LISiNOT Bioelettronica, Torino, Italy). Electrode grids recording from the wrist extensors were placed with their center positioned laterally above the extensor digitorum communis, and longitudinally above the muscle belly, while electrode grids over the wrist flexors were placed with their center positioned laterally above the flexor carpi radialis, and longitudinally above the muscle belly. A wrist band soaked in water served as common reference. The signals were amplified (EMGUSB2, OT Bioelettronica, Torino, Italy), band-pass filtered $(10-750 \mathrm{~Hz})$, and sampled at $2048 \mathrm{~Hz}$ by a 12 -bit A/D converter. Before placing the electrodes, the skin was lightly abraded with abrasive paste (Meditec-Pharma, Parma, Italy), cleansed with water and dried. The experiments were performed at Hospital Universitario '12 de Octubre', Madrid, Spain.

Wrist angular velocity was recorded with a pair of solidstate gyroscopes (Technaid S.L., Madrid, Spain) placed on the 
hand dorsum and the distal third of the forearm (dorsal side), following recommendations from previous studies [28, 29]. Data were sampled at $100 \mathrm{~Hz}$ by a 12 bit A/D converter and low-pass filtered (5th order Butterworth, cut-off frequency: $20 \mathrm{~Hz}$ ). Surface EMG and gyroscope recordings were synchronized using an external signal. Data were stored and analyzed offline using Matlab (The Mathworks, Natick MA, USA).

All recordings were performed while the patients were seated in a comfortable armchair. For ET patients, postural tremor was elicited by asking them to keep both hands outstretched with palms down, parallel to the ground, while the forearms were supported on an armrest. For PD patients, rest tremor was elicited by asking them to keep both arms relaxed and fully supported. Each recording lasted $4 \mathrm{~min}$.

\section{Experimental data analysis}

The convolution kernel compensation decomposition algorithm $[19,30,31]$ was used to extract single motor unit spike trains from the multi-channel surface EMG. As in previous studies of motor unit activity in tremor [32], only identified spike trains with pulse-to-noise ratio $\geqslant 26 \mathrm{~dB}$ (metric describing the decomposition accuracy; see [33] for details) was included in the analysis. For each muscle pair, a period of $30 \mathrm{~s}$ of the complete recording was selected for further analysis. Analysis intervals were selected to contain a high, stable level of tremor, including as many of the identified motor unit spike trains as possible. The cumulative spike train (CST) was an estimate of the neural drive to muscle and was defined as the algebraic sum of all single motor unit spike trains for each muscle. The ratio $H 2 / H 1$ was estimated for the CST as well as for each motor unit spike train, for the surface EMG recorded at the center of the high-density EMG matrix, and for the wrist angular velocity. To estimate $H 2 / H 1$, the tremor frequency was determined as the peak power frequency of the CST in the range $3-9 \mathrm{~Hz}$ (the typical range for the most common types of pathological tremors [2]). We verified that there was no spectral peak at half the peak frequency by visual inspection, to ensure that the second harmonic was not misclassified as the tremor frequency. The power at the tremor frequency $(H 1)$ was calculated as the integral of the smoothed power spectrum in a $\pm 0.5 \mathrm{~Hz}$ interval around the tremor frequency. The power at the second harmonic was estimated in a similar way at double the tremor frequency, but was corrected by subtracting the average power in two intervals with a width of $1 \mathrm{~Hz}$ centered $1.5 \mathrm{~Hz}$ above and below twice the tremor frequency, respectively (see figure 7 , Results). In this way, the continuous spectrum (equation (5)) that elevated the baseline power from frequencies near or higher than $H 2$ did not bias $H 2 / H 1$ towards higher values.

The individual tremor bursts were identified based on the zero-crossings of the derivative of the low-pass CST (5th order Butterworth; cut-off: $10 \mathrm{~Hz}$ ). For each burst, the timing (beginning, center, and end) was defined as the $20 \%$ quantile, median, and $80 \%$ quantile of the discharge times of the CST within the burst. Burst duration was calculated as the duration from the beginning to the end of the burst and was normalized

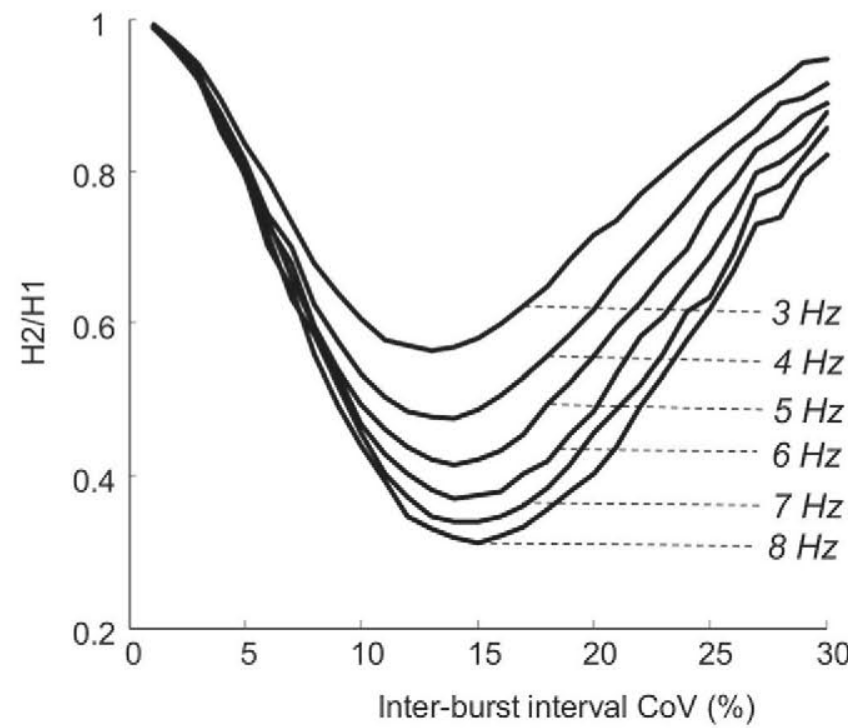

Figure 2. Influence of tremor frequency on the relation between $\mathrm{H} 2 /$ $H 1$ and inter-burst interval variability.

to the tremor period. Based on the identified burst centers, the $\mathrm{CoV}$ for the inter-burst intervals was also computed. For the single motor units, inter-burst variability was estimated from the single motor unit spike trains in the same way, and the average inter-spike interval within each burst was calculated.

Finally, the compound shape of the motor unit action potentials was estimated by spike-triggered averaging using the spike times of all motor units. In this way, the average motor unit action potential was estimated and its Fourier transform was computed to derive the filtering effect on the neural drive to the muscle (see equation (13)). The extraction of the average action potential was performed for IED of 8, 16 and $24 \mathrm{~mm}$ to assess the generalizability of its filter. Due to the high levels of motor unit short-term synchronization related to tremor, spike-triggered averaging could not be employed to accurately obtain the shapes of the motor unit twitches [34] to estimate the mechanical filters imposed on the neural drive. This filtering effect, however, has been quantified in several previous studies $[35,36]$.

\section{Statistical analysis}

For the single motor units and the CST, the experimental values of $H 2 / H 1$ were compared to the analytical predictions and the error between the measures and the predictions was calculated. The rectified errors across the different analytical models (doublet versus triplet for single motor unit spike trains and square pulse or sinusoidal burst approximation for the CST) were compared using the Kruskal-Wallis test. The comparison was made using the analytical models based on the nearest-integer tremor frequency (figure 2) compared to the experimental tremor frequency for each CST. For the single motor units, the comparison was made with the analytical models based on the average tremor frequency for each patient group, as the experimental $H 2 / H 1$ values were compiled across patients. Linear regression was used to determine the correlation between $H 2 / H 1$ and the inter-burst variability 
derived from the CST and the single motor unit spike trains, as well as the correlations between $H 2 / H 1$ of the CST, of surface EMG and of wrist angular velocity. F-test was used to determine significance of the linear relations. Finally, differences in tremor frequency, burst durations, and inter-burst interval variability were evaluated using Student's t-tests. For all tests, the level for significance was set to $p<0.05$.

To test the classification accuracy of the ratio $H 2 / H 1$ and other parameters with respect to differentiating patients with ET and PD, the average value across all muscles was obtained for each subject. Next, the value of this ratio that divided all samples into two groups with the optimal separation between ET and PD (lowest number of false classifications) was identified.

\section{Results}

The results are divided in two main parts. First, the analytical results present the foundation for how the temporal characteristics of one neural oscillatory network determine the amplitude of the tremor harmonics. The temporal characteristics included the variability in the interval between subsequent tremor bursts (inter-burst interval) and the width of the tremor burst. This was done considering single motor unit spike trains (accounting for the commonly observed behavior of doublet and triplet discharges [21, 22, 37]) as well as approximations of the compound output of the entire motor unit population (neural drive to the muscle). In this part and throughout the manuscript we define the ratio $H 2 / H 1$ as the power of the second harmonic of the oscillatory component of the neural drive to muscles in tremor relative to that of the first harmonics. In addition, this part includes a numerical estimation of the bias in the predicted values of $H 2 / H 1$ introduced by the presence of a second neural oscillator working at twice the tremor frequency projecting to the motor neurons.

In the second part, we present experimental validation from 22 tremor patients, from which to wrist angular velocity and high-density surface EMG were recorded. Trains of motor unit action potentials were identified from the EMG using the CKC $[19,30,31]$ decomposition algorithm and the neural drive to the muscles was defined as the ensemble of discharge timings of the motor unit pool innervating the muscle. This was estimated by summing their discharge times of each train of motor unit action potentials for each muscle in a CST [38].

\section{Analytical and simulated results}

The tremorogenic neural drive was approximated analytically as the convolution between a variable spike train at the tremor frequency (burst centers) and the shape of the tremor burst. While a perfectly regular series of tremor bursts would imply equal amplitude of all harmonics $(H 2 / H 1=1$; equation (3)), variability in the timing of the tremor bursts imposes a lowpass filter to the neural drive and thereby a reduction in $H_{2} /$ $H 1$ (figures $1(\mathrm{~A})$ and (B); equation (5)). For the shape of the burst in the neural drive, we considered a square wave (implying that all motor unit action potentials were evenly distributed in the tremor burst) and a truncated sine wave (implying a higher density of motor unit action potentials at the center of the burst), that in a similar way implied a filter to the underlying spike train describing the burst centers, thereby further affecting $H 2 / H 1$ (figures $1(\mathrm{C})-(\mathrm{F})$; equations (10) and (11)). As will be demonstrated below, this filter depended on the duration of burst. When considering the single motor unit spike train, the shape of the burst was defined as either a doublet (two spikes with short interval; equations (6) and (7)) or a triplet (equivalent for three spikes; equations (6) and (7)), whose filters depended on the interval between the spikes of the doublet/triplet. Due to the approximation of the neural drive as the convolution between the timing of the burst centers and the burst shape, the combined effect of both on the ratio $H 2 / H 1$ was obtained by multiplication in the frequency domain (equations (8)-(11)).

As a consequence of these filtering effects, the relation predicting the impact of the inter-burst interval variability on $H 2 / H 1$ depended on tremor frequency (figure 2). Across all frequencies, $H 2 / H 1$ was close to 1 when no variability was present and the minimum value was obtained at a $\mathrm{CoV}$ of approximately $15 \%$. At higher levels of variability $(<25 \%)$, $H 2 / H 1$ again increased, reflecting that the so-called line spectrum (see Methods) in these cases reached 0 at frequencies below the tremor frequency (see figure 1(B)).

In the case of a tremor frequency of $6 \mathrm{~Hz}$, the relation between the variability of the inter-burst intervals, withinburst inter-spike interval (for doublets and triplets, respectively), and $H 2 / H 1$ for single motor unit spike trains is depicted in figure 3 . The two relations were relatively similar, but differed in the gradient of $H 2 / H 1$ with respect to changes in the inter-burst interval. For both relations, values of $H 2 / H 1$ near 1 were obtained at brief inter-spike intervals and at low inter-burst variability. Furthermore, high values of this ratio could be obtained at very high levels of inter-burst variability $(\sim 30 \%)$, but as it will be shown below, the experimental results indicated that these levels of variability were not realistic in practice.

In a similar way, the relation between the variability of the inter-burst intervals, the burst duration (normalized to the tremor period), and $H 2 / H 1$ could be predicted for tremor burst approximated by the square pulse or the sine wave, using equations (10) and (11). Figure 4 shows these relations. Similarly to the relations for the single motor unit spike train (figure 3), high ratios $H 2 / H 1$ were obtained for regular and short tremor bursts. The primary difference across the two approximations for the tremor burst was that $H 2 / H 1$ approached 0 for the square pulse (figure 4(A)), whereas it stabilized at higher values $(0.2-0.5)$ for irregular, long bursts, when the sinusoidal approximation was used (figure 4(B)).

Figure 5 shows the results of the numerical simulations predicting the impact on $H 2 / H 1$ of neural oscillations at twice the tremor frequency superimposed on the tremorogenic neural drive. The bursts in the neural drive were approximated as square pulses and the relative amplitude and phase of the two oscillating signals was systematically varied. The 

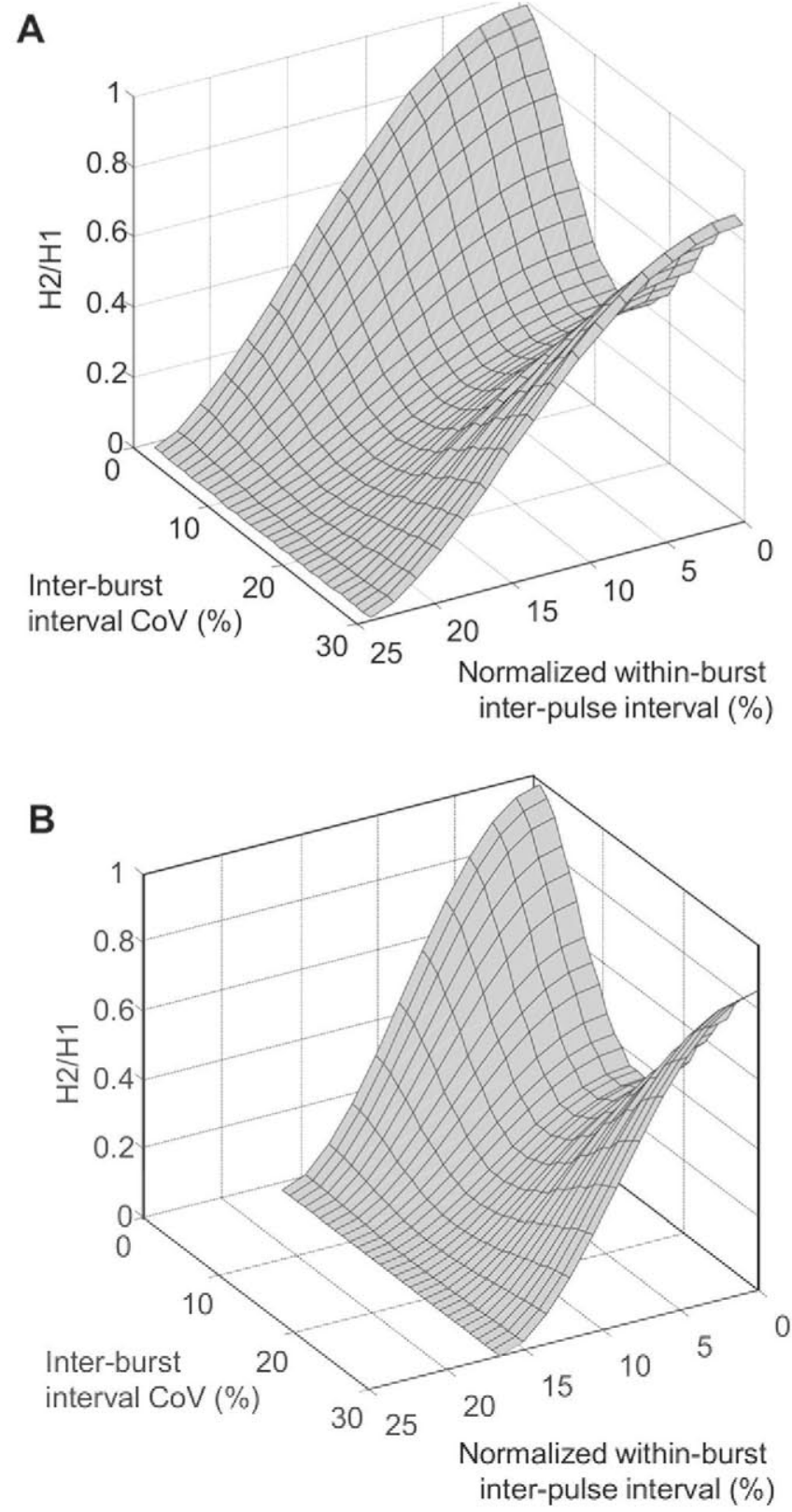

Figure 3. The analytically predicted relation between $H 2 / H 1$, tremor inter-burst interval variability and normalized inter-spike interval. This relation is depicted for doublets (A) and for triplets (B). In both illustrations, $6 \mathrm{~Hz}$ tremor was assumed.

outcome was the relative change in $H 2 / H 1$ (gain) induced by the second oscillator with respect to conditions with oscillations at the tremor frequency only across the different conditions. When the trains of square pulses were perfectly regular (figure $5(\mathrm{~A})$ ), the gain of $H 2 / H 1$ was highly phase dependent. In-phase timing implied an increase in $H 2 / H 1$ and vice-versa for out-of-phase timing. This was consistent with the analytical results, as in-phase timing reflects a higher amplitude of the neural drive at the center of the bursts, which is similar to the sinusoidal burst-approximation, which generally exhibited higher $H 2 / H 1$ values than the square pulse approximation (figure 4). On the other hand, out-of-phase
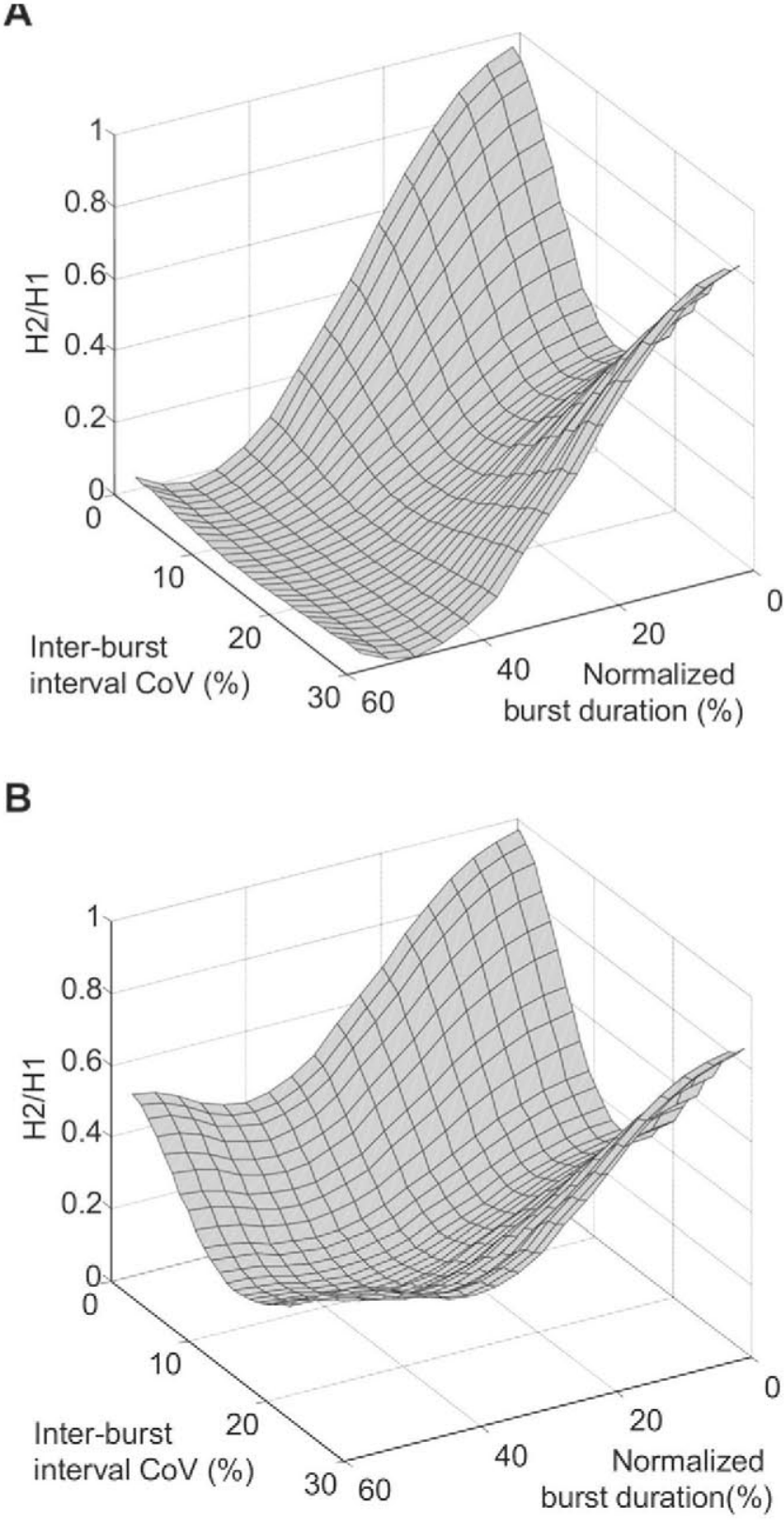

Figure 4. The analytically predicted relation between $H 2 / H 1$, tremor inter-burst interval variability and normalized burst duration. This relation is shown for tremor burst approximations by square pulses (A) or by a sinusoid (B). In both illustrations, $6 \mathrm{~Hz}$ tremor was assumed.

timing increases the burst duration, which analytically was related to a decrease in $H 2 / H 1$ (figure 4(A)). However, when irregularity in the timing of the burst centers was introduced, these interactions between the two series of pulses were lost and the superimposed oscillations served only to amplify the $H 2 / H 1$ ratio by an increase in the power at the frequency of $\mathrm{H} 2$ (figures 5(B) and (C)).

In summary, the theoretical analysis indicated that the ratio $H 2 / H 1$ can be predicted based on the temporal variability and the duration of the tremor bursts. The relation was non-linear and depended on a number of underlying assumptions, but in the realistic ranges of the parameters for 

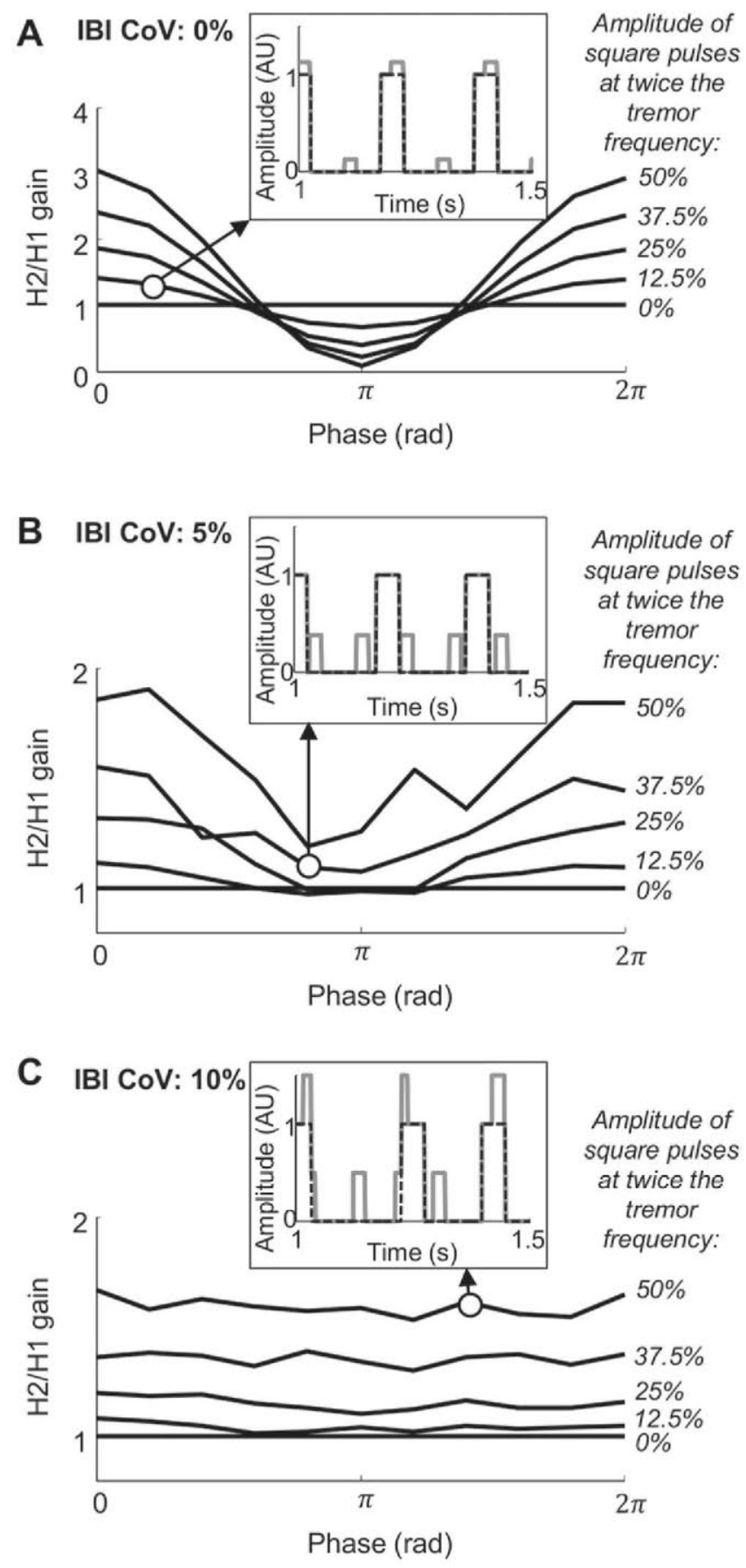

Figure 5. $H 2 / H 1$ in the presence of multiple oscillators. Numerical simulations of the bias in $H 2 / H 1$ introduced by additional oscillations in the neural drive at twice the tremor frequency for different levels of inter-burst interval variability (A) (C). The insets in each panel indicate the time-domain representation of the singleoscillator signal (black dashed line) and the compound signal including both oscillators (gray line). The gain of the $H 2 / H 1$ (relative difference with respect to the value obtained with oscillations at the tremor frequency only) was depicted as a function of the relative phase between the two oscillations ( $x$-axis) and the relative amplitude of the additional oscillations (black lines).

the motor unit behavior (see Experimental results below), a clear trend was present: short and regular tremorogenic bursts of motor unit activity implies values of the ratio $H 2 / H 1$ near 1 while this ratio decreases progressively as the duration and the temporal variability of the bursts increases. In the presence of a secondary oscillator at twice the tremor frequency, the power of the second harmonics $H 2$ was amplified to a degree depending on the strength of the oscillations, except if the two oscillators exhibited a perfect phase-locked behavior. Therefore, assuming two oscillators with even a low degree of independent temporal variability, the experimentally obtained values of $H 2 / H 1$ would be expected to be systematically higher than the analytically predicted values (figure 4).

\section{Experimental results}

The average tremor frequency was $5.9 \pm 1.0 \mathrm{~Hz}$ for the ET patients and $5.2 \pm 1.0 \mathrm{~Hz}$ for the PD patients (significantly different; $p=0.03$ ). On average, $8.3 \pm 5.1$ and $7.9 \pm 4.9$ motor unit spike trains per muscle were obtained from ET and PD patients, respectively (non-significant; $p=0.76$ ). Across all patients, the average number of single motor unit discharges per tremor burst was $2.2 \pm 0.7$ (range 1-5), but a very high variability was observed within each patient (average standard deviation: 0.72).

Figure 6 shows motor unit activity, surface EMG and the angular velocity from one representative ET patient. For this patient, the activity of 11 motor units was analyzed. These units clearly exhibited a burst-like behavior with a variable number of pulses per burst. For example, motor unit \#11 discharged 2-4 action potentials per burst. The tremorogenic behavior of the motor units was clearly reflected in the surface EMG and the wrist movement. Across all measurements, a clear spectral peak at the tremor frequency $(\sim 6 \mathrm{~Hz})$ was present. The amplitude of the first harmonic relative to the amplitude of the second harmonic (H2/H1), however, varied considerably across the modalities, according to the expected filtering effect on the neural drive to the muscle.

Considering the neural drive to the muscles, figure 7 depicts the power spectrum of the CST for one ET and one PD patient along with the characteristics of the motor unit population behavior (tremor burst width and inter-burst interval). As predicted by the analytical derivations, the patient (PD) with short tremor bursts (12.9\%; figure 7(B)) and with low variability of the inter-burst intervals $(\mathrm{CoV}=3.4 \%$; figure $7(\mathrm{C})$ ) had a high $H 2 / H 1$ ratio $(0.46$; figure $7(\mathrm{~A})$ ). In comparison, the $H 2 / H 1$ of the other patient (ET) was much lower $(0.03$; figure 7(D)), which is in accordance with the long tremor burst widths $(54.5 \%$; figure $7(\mathrm{E}))$ and highly variable inter-burst intervals $(\mathrm{CoV}=19.7 \%$; figure $7(\mathrm{~F}))$. This qualitative agreement with the theoretical derivations was quantitatively analyzed in all subjects, as depicted in figure 8. Figure $8(\mathrm{~A})$ presents the experimental association between variability and duration in the tremor oscillations and the ratio $H 2 / H 1$ in the neural drive (CST). Considering all patients, the non-rectified errors between the analytical prediction (assuming one oscillator approximated by square pulses) and the experimentally obtained values were not statistically different from zero (average values: PD: $-0.02, p=0.68$; ET: $-0.03, p=0.18$ ). Importantly, this suggests that a systematic bias in $H 2 / H 1$ towards higher values, as would be the 
A

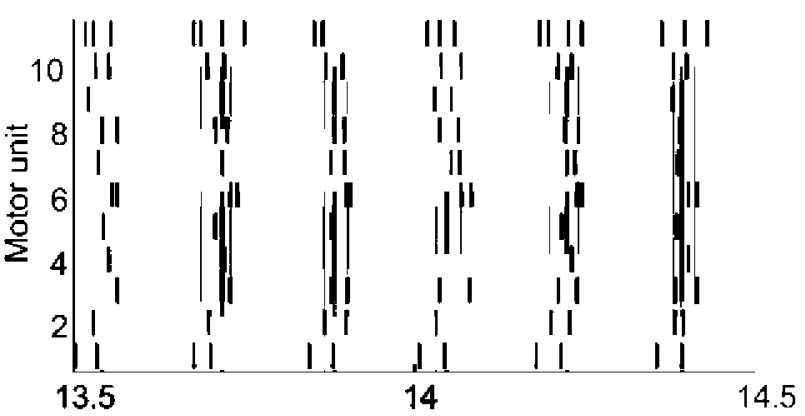

C

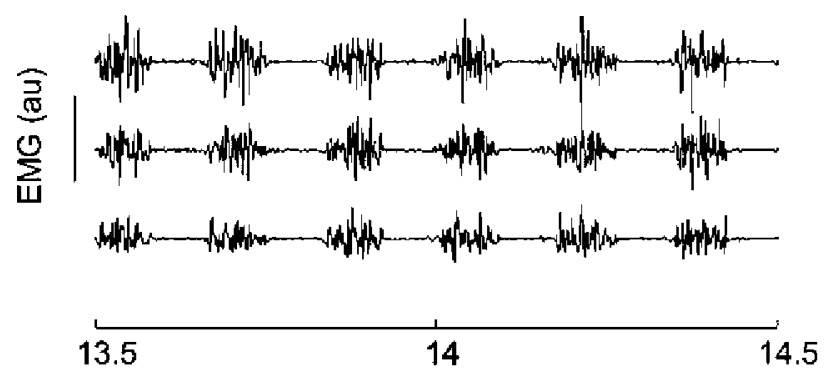

$\mathbf{E}$

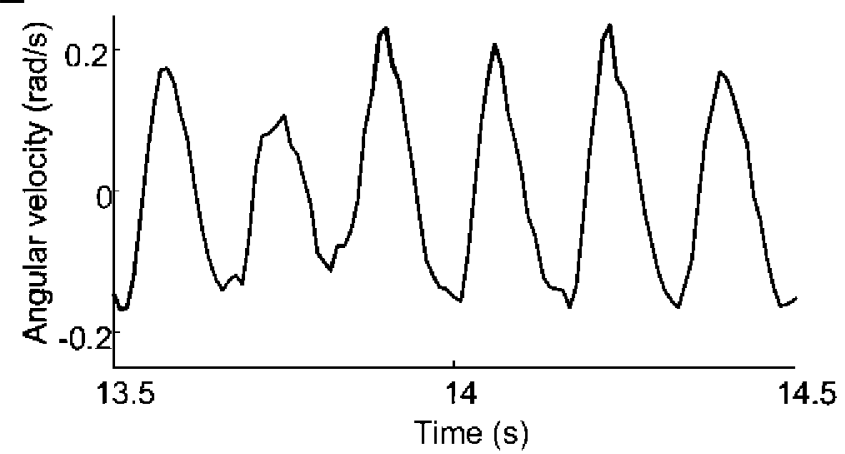

B

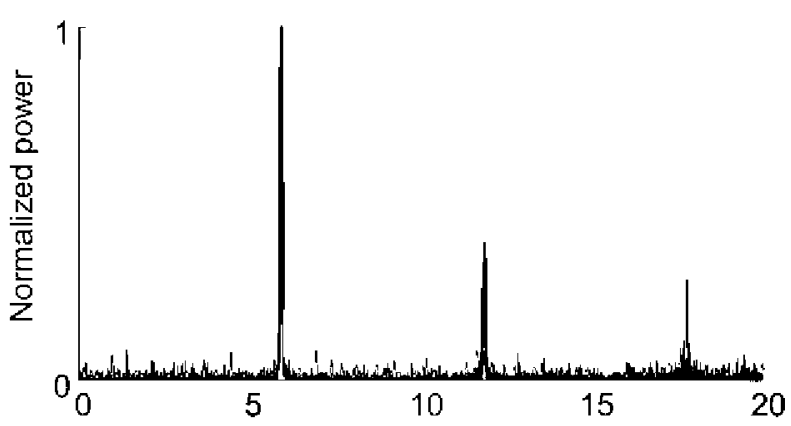

D

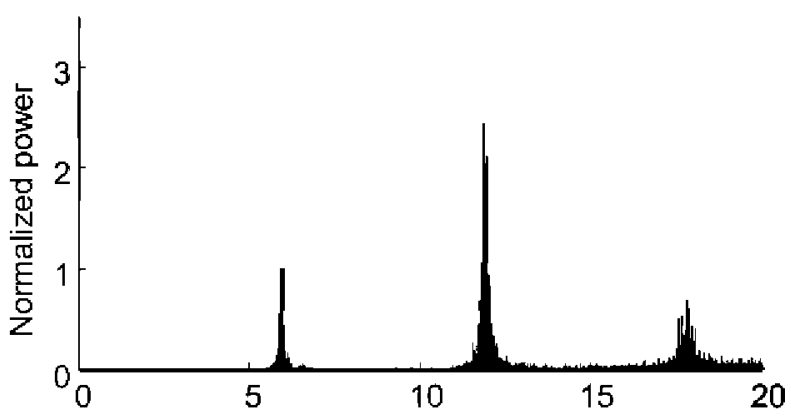

$\mathbf{F}$

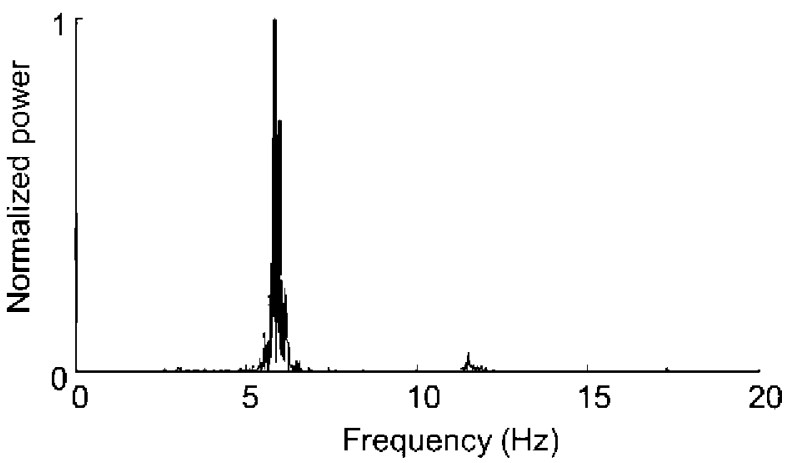

Figure 6. Tremor recordings in the spike train, the EMG and the movement. Representative data from one tremor patient showing time- and frequency-domain representations of motor unit activity (A),(B), surface EMG (C), (D) and wrist angular velocity (E), (F). 11 motor unit spike trains from the wrist extensor of the left arm were identified (A). Thick gray lines indicate the center of each tremor burst and the thin gray lines estimated beginning and end of the burst ( $20 \%$ and $80 \%$ quantile of all discharge times of the burst). A clear spectral peak at the tremor frequency $(6 \mathrm{~Hz})$ was present in the power spectra of all single motor unit spike trains. Surface EMG for three of the 59 channels recorded from the wrist extensor of the left arm (C) and the wrist angular velocity of the left arm (E) clearly exhibited tremorogenic behavior as indicated by their power spectra (D), (F).

consequence of the presence of a second oscillator at twice the tremor frequency (figure 5), was not found.

Considering the two patient groups separately, the values of $H 2 / H 1$ were significantly higher for PD patients $(0.26 \pm 0.23$ (PD) versus $0.09 \pm 0.13$ (ET); $p=0.0004$; figure $8(\mathrm{C})$ ). For ET patients, however, $H 2 / H 1$ was significantly lower for the extensor muscles (the muscle responsible for maintaining the wrist posture in the task) than for the flexors (median values: $0.04 \%$ versus $0.13 \%$; $p=0.02$ ). There was no cross-muscle difference for PD patients (median values: $0.24 \%$ versus $0.26 \% ; p=0.41$ ). By applying a threshold of 0.09 to the mean value of $H 2 / H 1$ for each patient, the two patient groups were classified with an accuracy of $86 \%$. In this way, three ET patients were misclassified (higher values of $H 2 / H 1>0.09$ ), while all patients with PD complied with this threshold. In accordance with the difference in $H 2 / H 1$ across ET and PD patients, the average burst duration was shorter $(29.2 \pm 17.2 \%$ (PD) versus $40.2 \pm 13.5 \%$ (ET); $p=0.005)$ and the mean inter-burst interval variability was lower (13.6 $\pm 6.3 \%$ (PD) versus $17.8 \pm 6.6 \%$ (ET); $p=0.009$ ) for the PD patients. The classification accuracy of these two variables, however, was lower than for $H 2 / H 1$ (Normalized burst duration: $68.1 \%$, optimal threshold: $18 \%$; Inter-burst interval variability: $66.7 \%$, optimal threshold $21 \%$ ). Similarly, tremor frequency had an classification accuracy of $65.2 \%$ (optimal threshold: $5.6 \mathrm{~Hz}$ ). Across all patients, the distribution of $H 2 / H 1$ as a function of burst duration and 
A

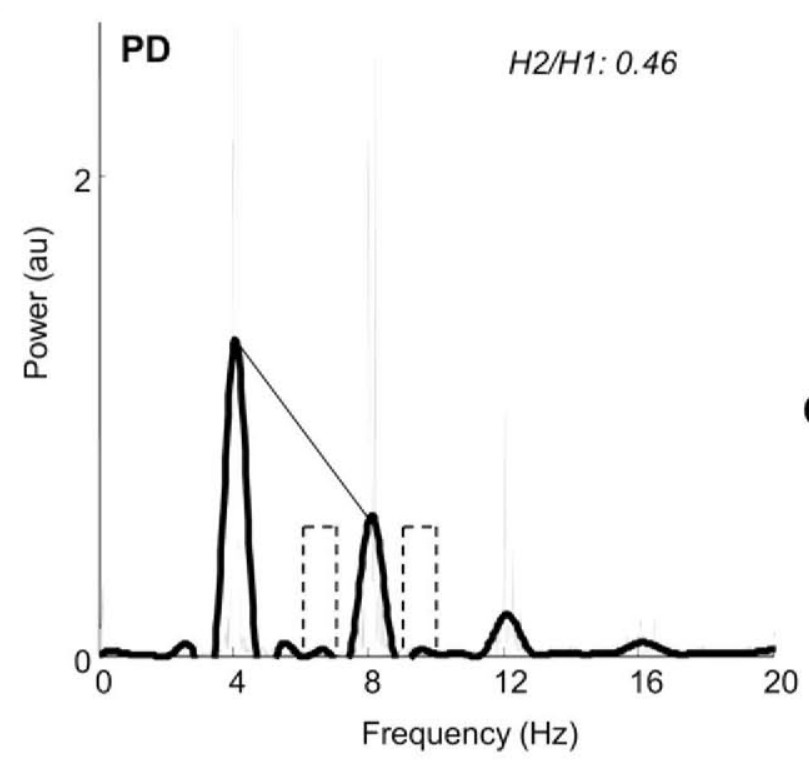

D

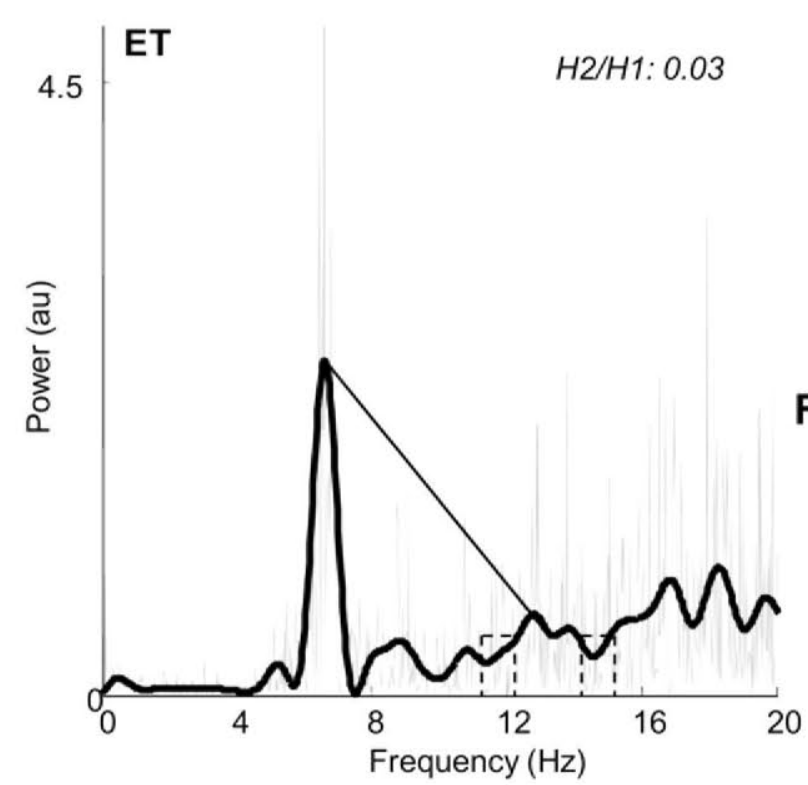

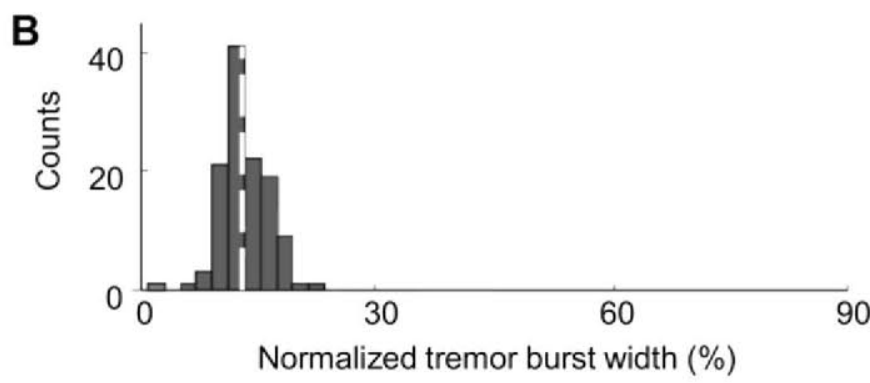

C

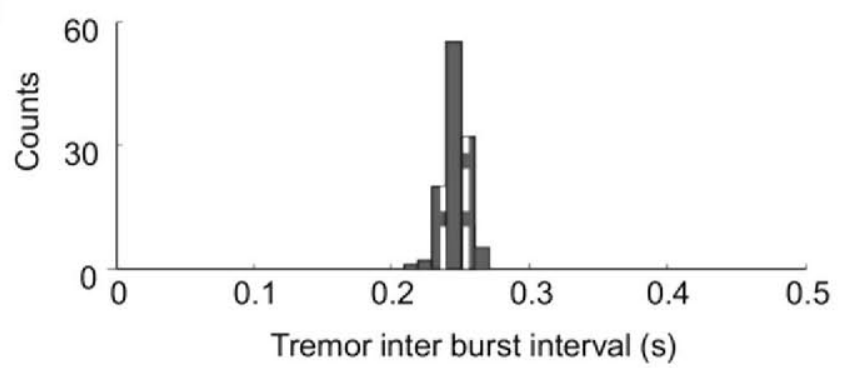

E

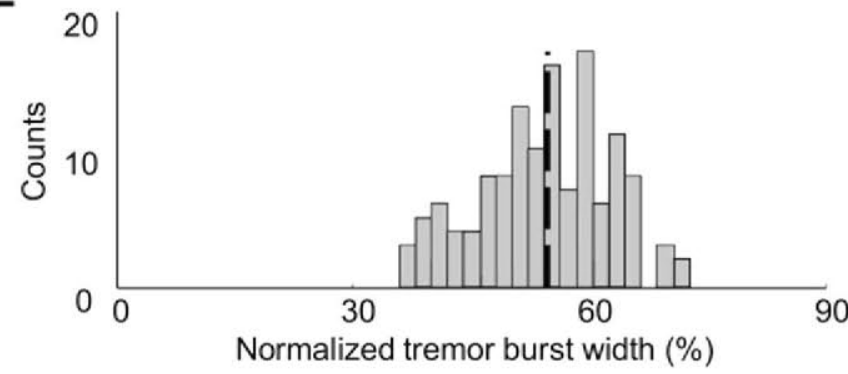

$\mathrm{F}$

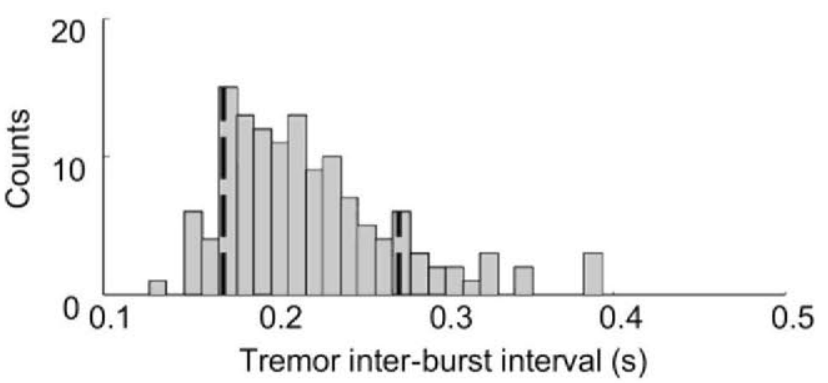

Figure 7. Power spectrum of neural drive and its temporal characteristics. Smoothed power spectra of the CST from one muscle in one patient with PD (A) and one patient with ET (D). Light gray lines indicate the raw power spectra. In these two muscles, spike trains from 12 to 6 motor units were identified, respectively. The power at the harmonics $(H 1$ and $H 2)$ are indicated by the line. The mean power in the frequency range indicated by the dashed areas was subtracted from the power at $H 2$ in order to compensate for high baseline power in that range. Panels B and E show histograms of the normalized tremor burst widths for each burst of the two muscles estimated from the CST. Here, the dashed line represents the mean burst width. Panels C and F show histograms of the tremor inter-burst intervals for each burst of the two muscles. Here, the white dashed lines represent the mean inter-burst interval \pm its standard deviation.

variability (figure $8(\mathrm{~A})$ ) corresponded well to that predicted analytically for square pulses (see figure 4(A)). Here, the median rectified error (PD: 0.14; ET: 0.06, both in units of the ratio $H 2 / H 1$ ) was significantly smaller than the error related to the approximation based on the sinusoid function $(p<0.0001$ (ET) and $p=0.049$ (PD)). Visual inspection of smoothed spike histograms for the tremor bursts (results not shown) confirmed that action potentials tended to be uniformily distributed across the burst. For the $H 2 / H 1$ obtained from the power spectrum of single motor unit spike trains, six motor units (out of 654) with $H 2 / H 1>10$ were considered outliers and were not included in the analysis. As predicted analytically, high $H 2 / H 1$ values were attained for motor units exhibiting short inter-spike intervals with low variability of the inter-burst intervals (figure 8(D)). Only a small proportion of the motor units exhibited inter-burst variability $>30 \%(n=28)$. For the analysis of the error with respect to the analytical predictions, only motor units with average inter-spike intervals $<25 \%$ for doublets and $<17 \%$ for triplets (as defined in figure 3 ) were considered, as motor unit spike trains with longer inter-spike intervals usually exhibited very low tremorogenic behavior. For both patient 
A

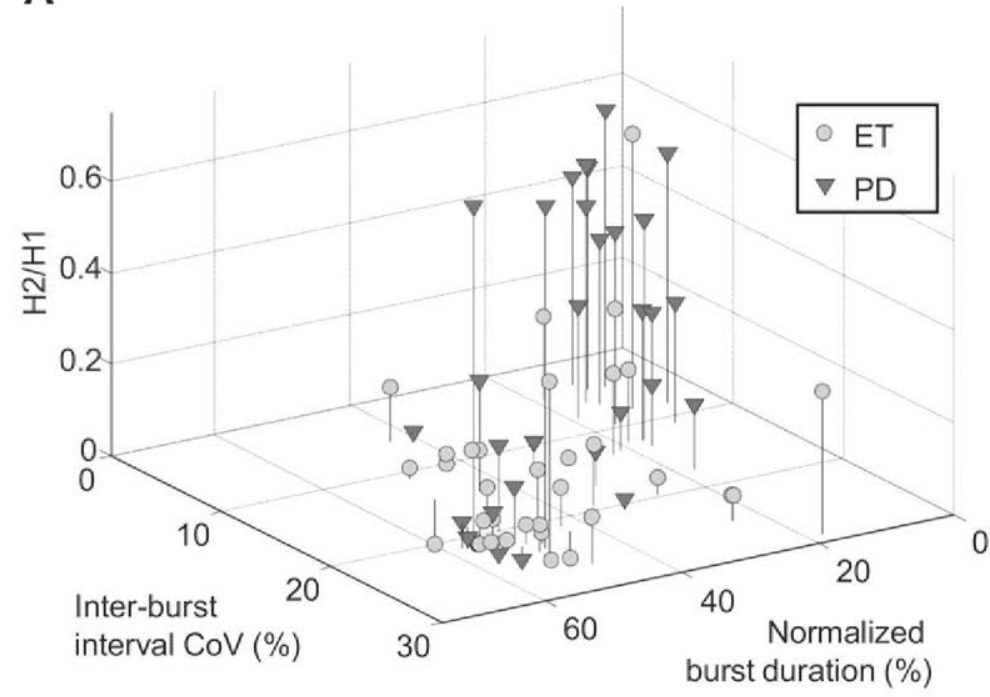

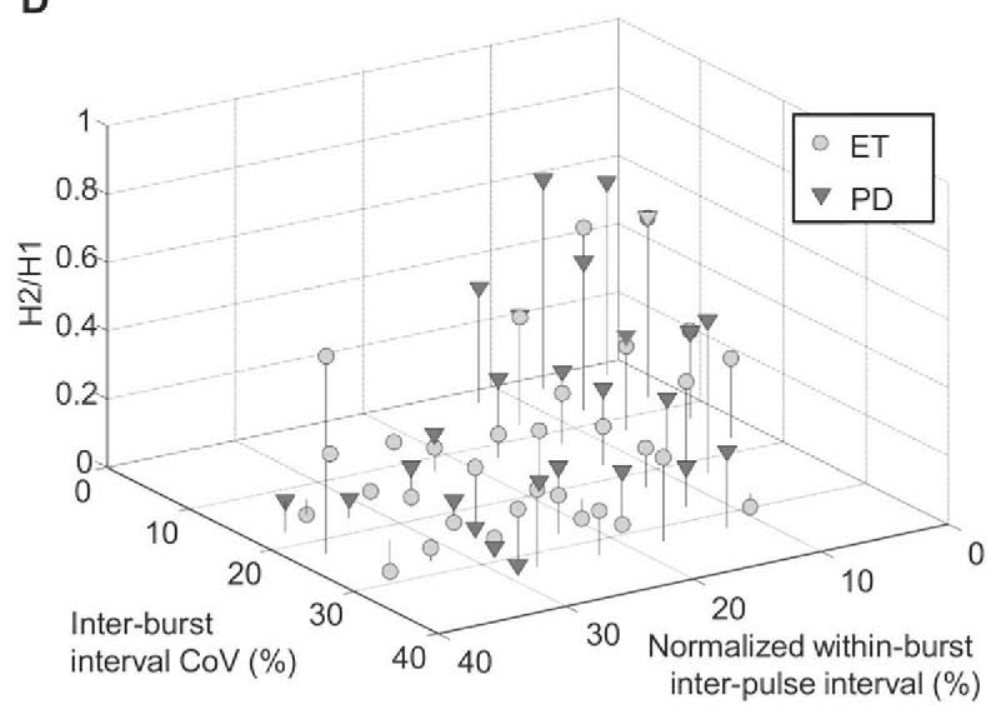

B

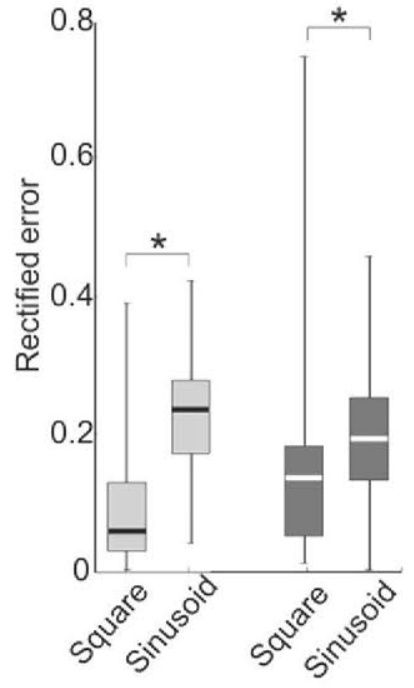

E

C

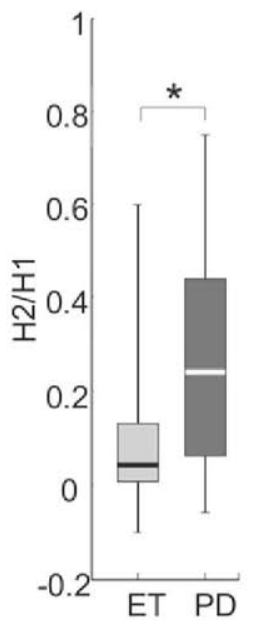

$\mathbf{F}$

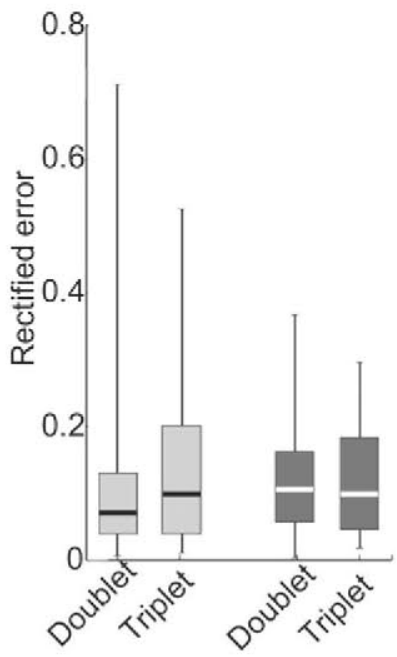

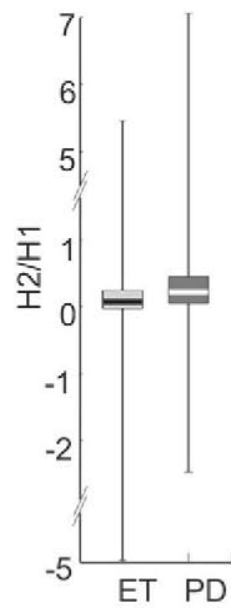

Figure 8. Comparison of predicted and experimental $H 2 / H 1$. The values of $H 2 / H 1$ for the CST of all muscles from all subjects depicted as a function of the coefficient of variation of the inter-burst interval and the normalized burst duration (A), the rectified error between the experimental results and the analytical predictions for the approximations of the tremor bursts by a square wave and the square wave multiplied by a sinusoid (B) and the distribution of $H 2 / H 1$ for ET and PD (C). The approximation based on a square wave yielded significantly lower rectified errors for both patient groups. Light gray circles (A), (D) and light gray boxes (B), (C), (E), (F) indicate ET patients whereas dark gray triangles (A), (D) and dark gray boxes (B), (C), (E), (F) indicate PD. In panels A and D, vertical lines indicate the location of each data-point on the $x-y$ plane. $H 2 / H 1$ for the single motor unit spike trains of all muscles for all subjects as a function of the coefficient of variation of the inter-burst interval and the normalized inter-pulse interval (D). The values of $H 2 / H 1$ are averaged in groups of motor units with similar values on the $x$ - and $y$-axis. Groups with less than three motor units were excluded. The rectified error between the experimental results and the analytical predictions for the doublet and triplet discharges were not significantly different (E). In total, 186 motor units for doublets (with within-doublet inter-spike intervals $<25 \%$ ) and 115 motor units for triplets (with within-triplet inter-spike intervals $<17 \%$ ) were considered. Values of $H 2 / H 1$ across ET and PD were not statistically significant (F).

groups, median rectified errors for both doublet and triplet were relatively low $(<0.1$; figure $8(\mathrm{E}))$. There was no significant difference between the errors, which may be explained by the high within-unit variability in the number of action potentials per burst. Furthermore, although the PD patients again exhibited the highest average value of $H 2 / H 1$ there was no significant difference between the two patient groups when estimating this ratio using trains of single motor unit discharges $(0.28 \pm 0.74$ (PD) versus $0.19 \pm 1.5$ (ET); figure 8(F)).

\section{$\mathrm{H} 2 / \mathrm{H} 1$ of single motor units spike trains versus CST}

The values of $H 2 / H 1$ obtained from the CST were significantly correlated to those obtained from the single motor unit spike trains (figure 9(A)). However, a large variability in 
A

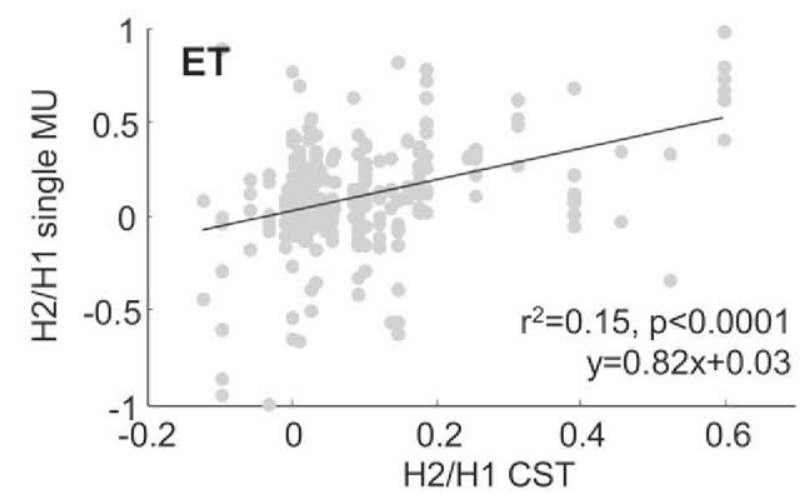

B

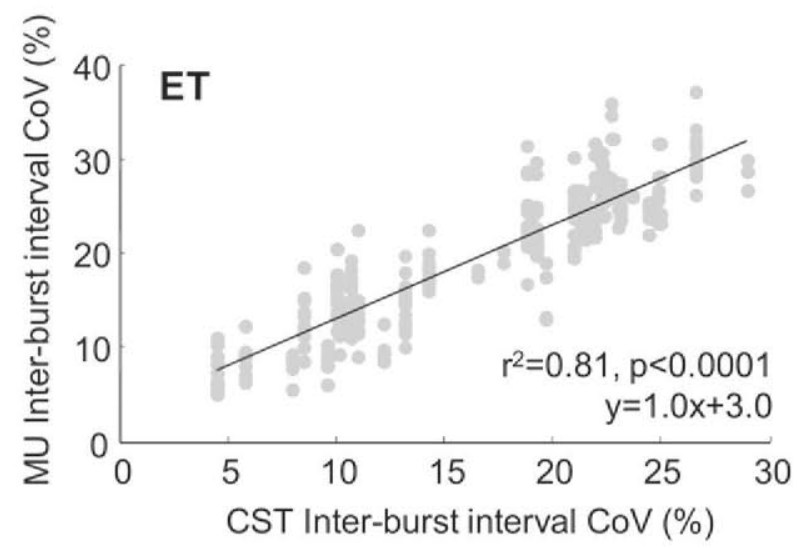

C

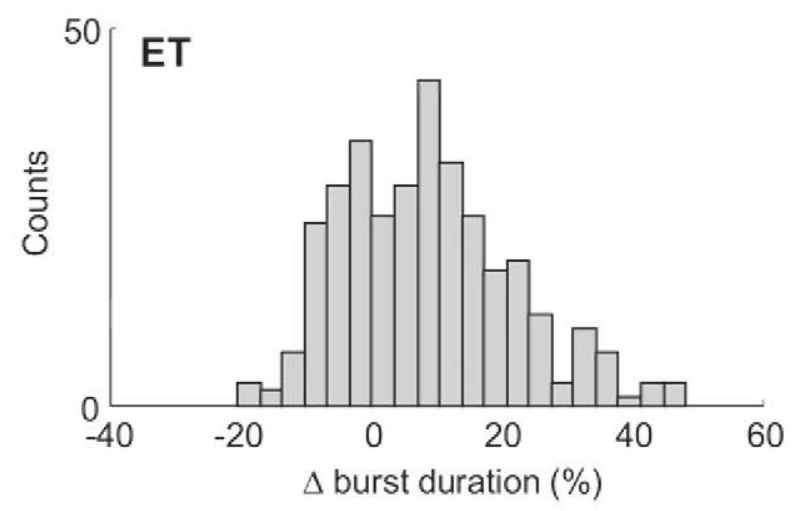

D

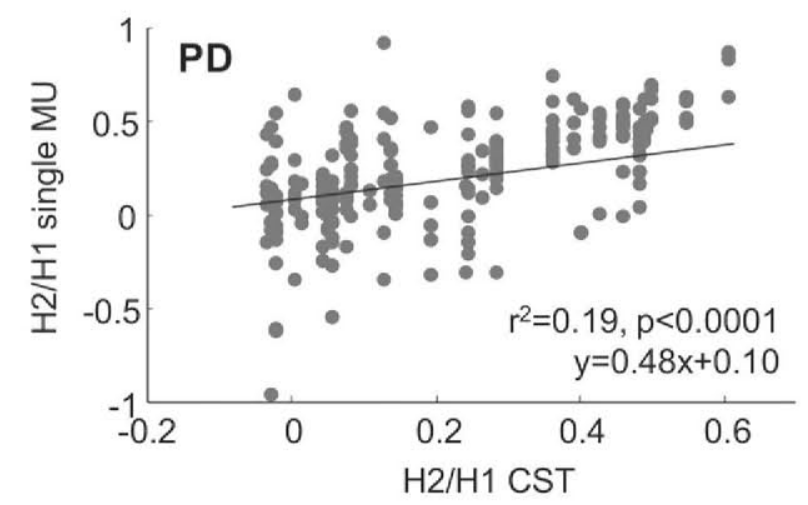

E

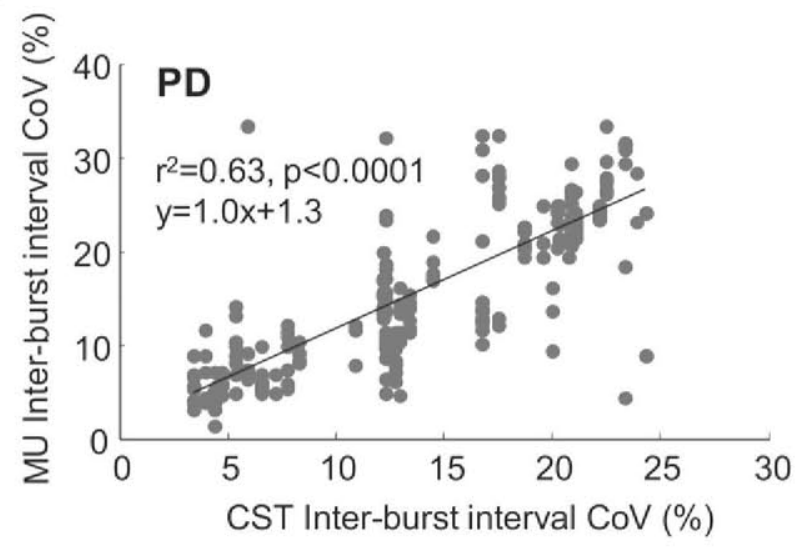

$\mathbf{F}$

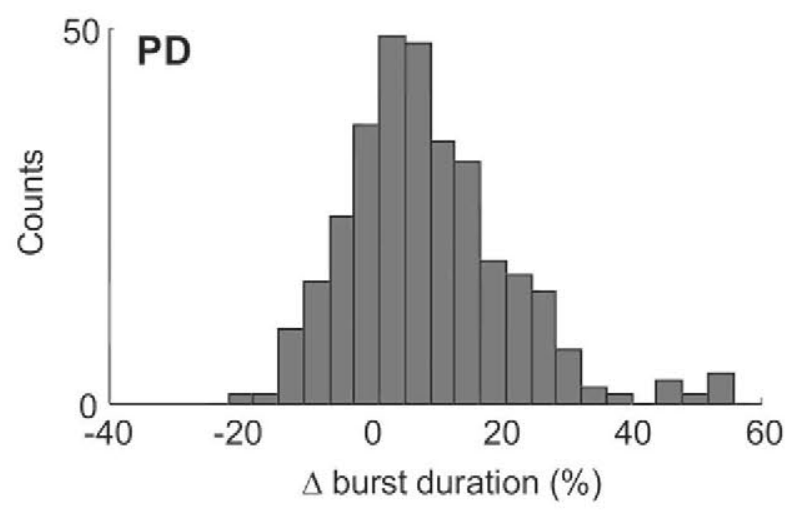

Figure 9. $H 2 / H 1$ from the CST and single motor unit spike trains. The correlation between $H 2 / H 1$ obtained from the CST and from single motor unit spike trains (A: ET; D: PD), the correlation between the inter-burst interval estimated from the CST and from the single motor unit spike trains (B: ET; E: PD), and the distribution of difference between the burst durations obtained from CST and single motor unit spike trains (C: ET; F: PD).

$H 2 / H 1$ within each motor unit population was observed, as indicated by the relatively low correlation coefficients (PD $r^{2}=0.19$; ET: $r^{2}=0.15$ ). The analytical derivations (figures 3 and 4) indicate that this discrepancy may be explained by differences in the variability of the inter-burst intervals between the single units and their population, or by differences in the estimation of the tremor burst duration in the case of the single motor unit action potentials and in the case of the whole identified motor unit population. The variability in the timing of the tremor burst centers in the single motor units and in the motor unit population was highly similar, indicating that this parameter is not the source of the variability (PD: $r^{2}=0.63$; ET: $r^{2}=0.81$; figures $9(\mathrm{~B})$ and (E)). Instead, a large variability in the estimation of the bursts' durations at the two levels analyzed (single motor unit and CST) was a more likely explanation (figures $9(\mathrm{C})$ and $(\mathrm{F})$ ). On average, the normalized tremor burst durations at the single motor unit level were only moderately shorter than those of the CST (ET: 
A

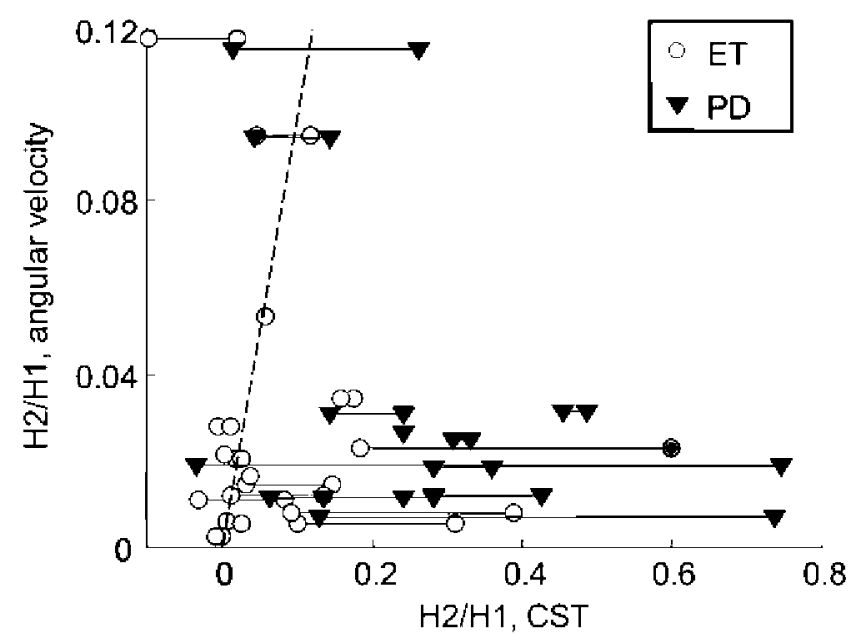

B

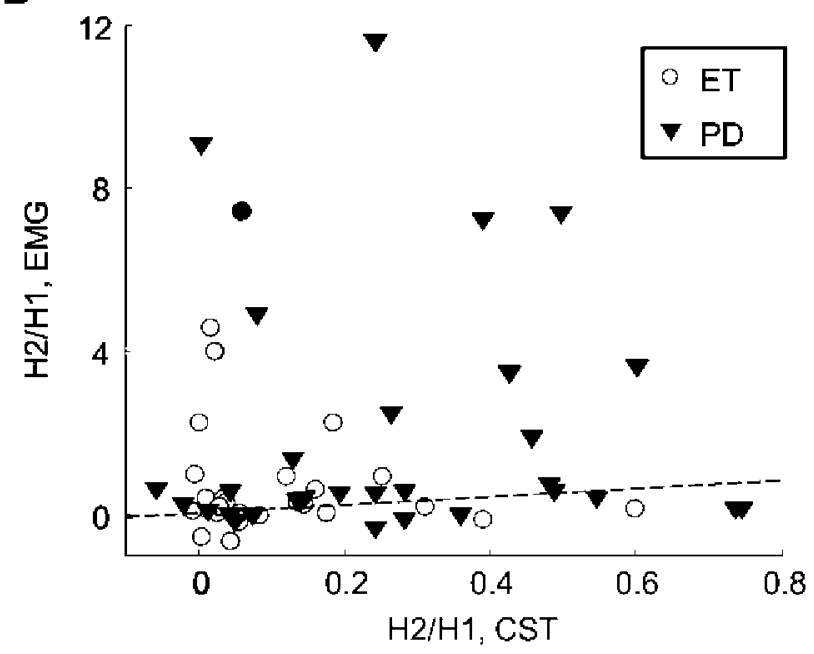

Figure 10. $H 2 / H 1$ from neural drive, EMG and movement. Values of $H 2 / H 1$ obtained from CST plotted against those obtained from wrist angular velocity (A) and from the surface EMG signal (B) for ET (light gray circles) and PD (dark gray triangles). The relations were not significant in any of the cases. In A, the horizontal lines indicate the range spanned by the values of $H 2 / H 1$ obtained from the CST of each of the two antagonist muscles affecting the wrist joint. In three cases the horizontal line is missing as the results from one of the muscles was excluded as less than three motor units was identified. In both panels, the dashed line represents the identity $x=y$.

8.8 percentage points; PD: 9.0 percentage points), but the standard deviations of the distribution were high (ET: 13.0 percentage points; PD: 12.4 percentage points).

\section{H2/H1 of inertial measurement and surface EMG versus CST}

There were no significant correlations between $H 2 / H 1$ obtained from the CST and those obtained from either the wrist angular velocity (figure $10(\mathrm{~A})$ ) or the surface EMG (figure 10(B)) for PD or ET patients. Even though the $H 2 / H 1$ was higher for PD patients than for ET patients when obtained from the angular velocity (PD: $0.033 \pm 0.034$; ET: $0.028 \pm 00.033$ ) or from the EMG (PD: $1.24 \pm 2.14$; ET:
$0.75 \pm 01.84$ ), these differences were not statistically significant for any of the two modalities. Across both patient groups, the values of $H 2 / H 1$ obtained from the angular velocity generally underestimated those from the CST. This effect can be predicted from the theoretical derivations (equation (13)) and the low-pass mechanisms of the forcegenerating and mechanical systems [39]. Conversely, $H 2 / H 1$ obtained from the surface EMG overestimated that of the CST in most cases. Also in this case, the effect is predicted by the theory and explained by the high-pass filtering characteristics of the shapes of the motor unit action potentials on the neural drive to the muscle. Figure 11 shows a representative action potential estimated by spike-triggered averaging and the filtering characteristics on the neural drive. The power spectra of all estimated compound action potentials implies a band-pass filter applied to the neural drive with the lower cut-off frequency at $45.9 \pm 37.3 \mathrm{~Hz}, 43.0 \pm 19.3 \mathrm{~Hz}$, and $43.1 \pm 31.6 \mathrm{~Hz}$ for IEDs of 8 (illustrated in figure 11(B)), 16 and $24 \mathrm{~mm}$ respectively. The large variability in this filtering effect (also illustrated by the light colored traces in figure 11(B)) evidently hinders the possibility of compensating for it in experimental cases.

\section{Discussion}

In this study we investigated the two hypotheses regarding the neural drive to muscles in tremor, that either one neural oscillator working at the tremor frequency, or the superimposition of two oscillators at the tremor frequency and twice that frequency, respectively, entrains the involuntary rhythmical limb movements. Using an analytical approach it was shown that a large range of values for the ratio $H 2 / H 1$ could be achieved within realistic ranges of tremor burst duration and variability, assuming a single oscillatory network. High values of the ratio $H 2 / H 1$ were achieved at highly regular, short tremor bursts, but the theoretical relation between these variables was non-linear and depended on tremor frequency (figure 2) and the statistical distribution of the action potentials within the tremor bursts (figure 4). Superimposing oscillations at twice the tremor frequency on the neural drive implied a systematic bias towards higher values of $H 2 / H 1$. Experimental data from 22 tremor patients showed that the values $H 2 / H 1$ estimated from the neural drive depended on the regularity of the neural drive as predicted analytically, with a low error between the theoretical and experimental results (figure 8).

This finding has implications for the understanding of the central oscillatory networks underlying pathological tremor and specifically the unresolved question of whether one or more central oscillatory networks are driving pathological tremors $[17,18]$. The low error between the analytically predicted and the experimentally obtained values of $H 2 / H 1$ indicated that the magnitudes of the first harmonic across all patients can be explained almost perfectly by only one oscillatory network with temporal properties that varies across patients. On the contrary, the fact that there was no systematic bias in the error between the analytical and experimental 
A

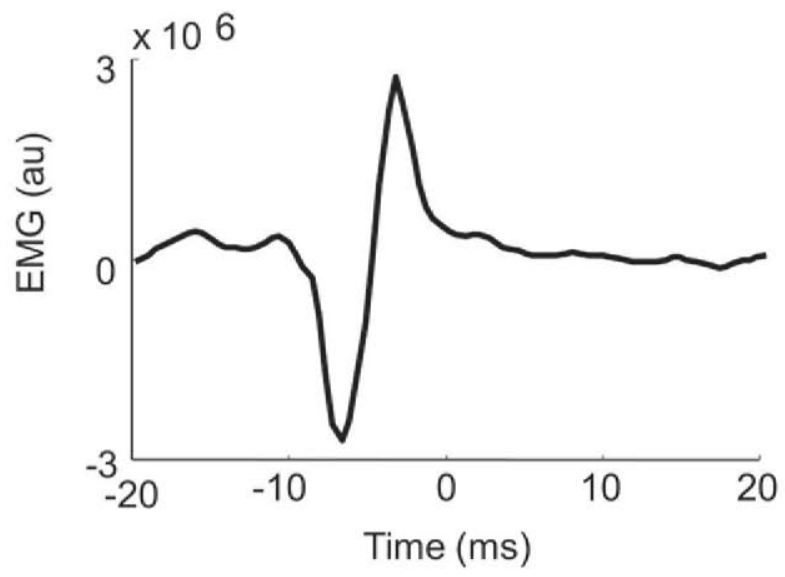

B

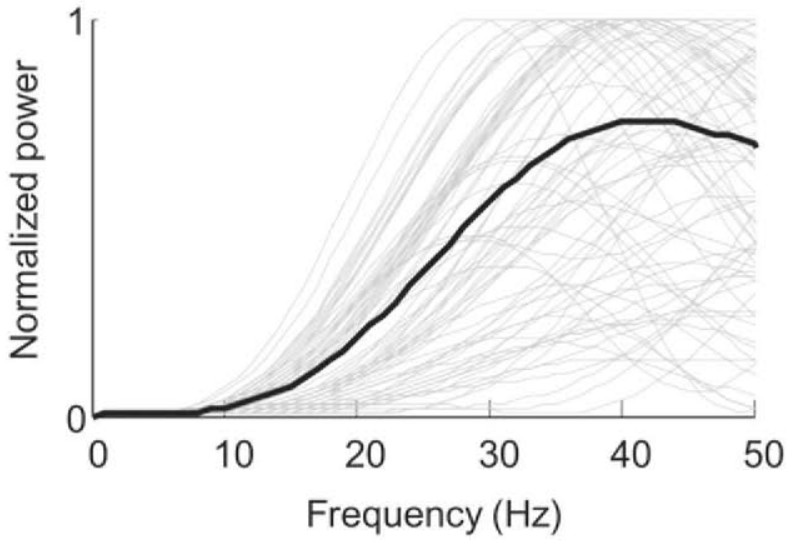

Figure 11. Filtering properties of EMG. Spike triggered average compound motor unit action potentials obtained from the single differential EMG signals with inter-electrode distance $8 \mathrm{~mm}$. Panel B shows the normalized power spectra of all motor unit action potentials. The bold black lines indicate the average power of all action potentials.

results across all measurements is incompatible with a neural drive to the muscles reflecting a superimposition of the output of two oscillators. Although the results cannot rule out the presence of a second neural oscillator at twice the tremor frequency projecting to the motor neurons, as previously indicated by analysis of EEG-EMG coherence [17], the findings suggest that its relative contribution to the output of the motor neurons (neural drive to the muscle) would in any case be low (figure 5). Alternatively, the relative timing between the two oscillations needs to be perfectly regular, which seems unlikely, given the experimental inter-burst interval variability (figure 8). One possible explanation for these conflicting findings could be that both oscillators are present in the descending contribution to the motor neuron input, but that other sources of synaptic input at the spinal level serve to amplify the component at the tremor frequency only. Although the contribution of afferent feedback in tremor has never been directly quantified, several observations indicate that it provides a substantial contribution to the amplitude of the tremor [37, 40-42]. Since feedback from muscle spindle receptors encodes muscle movement [43, 44] which is a low-passed filtered version of the neural drive [35, 39], the afferent input could be hypothesized to selectively amplify the low-frequency components of the tremorogenic descending drive, and thus the tremor frequency rather than input at twice that frequency.

A second important finding of the study was that systematic differences in regularity of the oscillations in the neural drive implied that the ratio $H 2 / H 1$ was different across ET and PD patients. As a direct consequence, the relative power of the tremor harmonics was significantly higher for patients with PD. This difference may in part be task-related (posture/rest), since the values of $H 2 / H 1$ in ET patients were higher for the flexor muscle (presumably relaxed during the postural task) than for the extensors (maintaining the posture against gravity). For both muscles, however, $H 2 / H 1$ was higher in the PD patients, indicating that the difference cannot be exclusively attributed to the task. Accordingly, this measure was able to correctly classify patient diagnosis in $86 \%$ of the cases, implying its value as a biomarker for tremor diagnosis although tests in larger number of patients are required to verify its classification accuracy. In addition, the results indicated that in order for $H 2 / H 1$ to reflect the temporal characteristics of the neural drive to the muscle, this ratio must be estimated based on the CST, the most direct in vivo estimate of the neural drive to muscle [38, 45]. In comparison, when this ratio was calculated from the power spectra of single motor unit spike trains it was more affected by random variability (noise) in the discharge times, implying very high within-muscle variability in $H 2 / H 1$. This noise was also reflected in a high variability in the number of discharges per tremor burst for each motor unit, and, consequently, also in high variability in the duration of the activity of the motor unit within each burst. Similarly, mechanical (gyroscope) and electrophysiological (EMG) measures of tremor introduced bias in the estimation of tremor regularity, which could be studied and predicted as a filtering effect on the neural drive. The high-pass filtering properties of the motor unit action potentials implied an attenuation of the power at the base tremor frequency with respect to the power at the first harmonic (figure 11). The large inter-subject variability in the high-pass properties of the MUAP (in particular, standard deviations in cut-off frequency of $\sim 30 \mathrm{~Hz}$ ) implied that the magnitude of this effect is difficult to predict and that the $\mathrm{H} 2 /$ $H 1$ derived from the EMG is therefore not suited for the estimation of the temporal tremor characteristics. The angular velocity, on the other hand, is the result of a low-passed filtered version of the neural drive due to the properties of the motor unit twitch [35, 39] and the viscoelastic mechanical properties of muscles [36, 46]. Furthermore, this low-pass filtering is highly pronounced in older adults, due to the agerelated slowing of muscle contractile speed [47]. In this way, the ratios derived from angular velocity and the EMG signal tended to under- and overestimate the value of $H 2 / H 1$ calculated from the neural drive, respectively (figure 10). Importantly, these biases showed little consistency across subjects, which implied there was no significant difference in $H 2 / H 1$ across ET and PD when estimated from either EMG or 
the angular velocity. In this way, the results indicate that the use of $H 2 / H 1$ as a diagnostic biomarker for tremor diagnosis is more accurate when using a direct measure of the neural drive (CST). It should, however be noted that it is possible that alternative recording configurations of EMG (electrode type, spatial filters, etc) and movement sensor may improve the estimation accuracy. The tremorogenic neural drive can be obtained in a non-invasive way, as demonstrated in this and in previous studies [19]. These results also suggest that the high classification accuracy of the non-normalized average power of the angular velocity harmonics reported in a previous study [11], may reflect task-dependent differences in tremor severity (reflected in the absolute power of the first harmonic) across the two patient groups rather than the underlying regularity of the oscillatory neural drive. Finally, the results suggested that the central network output is best approximated by a series of square pulses (figure 8) with variable temporal behavior depending on the underlying pathology, which may be relevant to e.g. simulations of pathological tremor using computational models (e.g. [42, 48]).

In conclusion, the results support the hypothesis that the oscillations in the output of the motor unit population in pathological tremor is driven by one oscillatory input at the tremor frequency and that the regularity of this input is different across ET and PD patients. For this reason, the relative amplitude of the second harmonic of the tremor frequency in the neural drive to the muscle is significantly different across the two patient groups, reflecting that the tremoric bursts of motor unit activity in PD are shorter and more regular compared to ET. The filtering process inherent in the transformation of the neural drive into movement or EMG bias the ratio $H 2 / H 1$, implying that when estimated from these measurements this ratio loses its diagnostic value.

\section{Acknowledgments}

The authors are grateful to Dr Juan Pablo Romero and Dr Julián Benito-León, Hospital Universitario '12 de Octubre', Madrid, Spain for organizing patient recruitment and for practical assistance during the experiments.

\section{References}

[1] Wenning G K, Kiechl S, Seppi K, Müller J, Högl B, Saletu M, Rungger G, Gasperi A, Willeit J and Poewe W 2005 Prevalence of movement disorders in men and women aged 50-89 years (Bruneck Study cohort): a population-based study Lancet Neurol. 4 815-20

[2] Deuschl G et al 1998 Consensus statement of the movement disorder society on tremor Mov. Disord. $132-23$

[3] Thenganatt M A and Louis E D 2012 Distinguishing essential tremor from Parkinson's disease: bedside tests and laboratory evaluations Expert Rev. Neurother. 12 687-96

[4] Benito-León J and Louis E D 2006 Essential tremor: emerging views of a common disorder Nat. Clin. Pract. Neurol. 2 $666-78$ quiz $2 p$ following 691
[5] Jain S, Lo S E and Louis E D 2006 Common misdiagnosis of a common neurological disorder: how are we misdiagnosing essential tremor? Arch. Neurol. $631100-4$

[6] Milanov I 2001 Electromyographic differentiation of tremors Clin. Neurophysiol. 112 1626-32

[7] Palmes P, Ang W T, Widjaja F, Tan L C S and Au W L 2010 Pattern mining of multichannel sEMG for tremor classification IEEE Trans. Biomed. Eng. 57 2795-805

[8] Jakubowski J, Kwiatos K, Chwaleba A and Osowski S 2002 Higher order statistics and neural network for tremor recognition IEEE Trans. Biomed. Eng. 49 152-9

[9] Hossen A, Muthuraman M, Al-Hakim Z, Raethjen J, Deuschl G and Heute U 2013 Discrimination of Parkinsonian tremor from essential tremor using statistical signal characterization of the spectrum of accelerometer signal Biomed. Mater. Eng. 23 513-31

[10] Deuschl G, Lauk M and Timmer J 1995 Tremor classification and tremor time series analysis Chaos $\mathbf{5} 48-51$

[11] Muthuraman M, Hossen A, Heute U, Deuschl G and Raethjen J 2011 A new diagnostic test to distinguish tremulous Parkinson's disease from advanced essential tremor Mov. Disord. $261548-52$

[12] Groznik V, Guid M, Sadikov A, Mozina M, Georgiev D, Kragelj V, Ribaric S, Pirtosek Z and Bratko I 2013 Elicitation of neurological knowledge with argument-based machine learning Artif. Intell. Med. 57 133-44

[13] McAuley J H, Rothwell J C and Marsden C D 1997 Frequency peaks of tremor, muscle vibration, and electromyographic activity at $10 \mathrm{~Hz}, 20 \mathrm{~Hz}$ and $40 \mathrm{~Hz}$ during human finger muscle contraction may reflect rythmicities of central neural firing Exp. Brain Res. 114 525-41

[14] Heckman C J and Enoka R M 2004 Physiology of the motor neuron and the motor unit Neurophysiology of Motor Neuron Diseases (Handbook of Clinical Neurophysiology) vol 4 ( ed A Eisen (Amsterdam: Elsevier) pp 119-47

[15] Helmich R C, Toni I, Deuschl G and Bloem B R 2013 The pathophysiology of essential tremor and Parkinson's tremor Curr. Neurol. Neurosci. Rep. 13378

[16] Louis E D, Babij R, Cortés E, Vonsattel J-P G and Faust P L 2013 The inferior olivary nucleus: a postmortem study of essential tremor cases versus controls Mov. Disord. 28 $779-86$

[17] Raethjen J, Govindan R B, Muthuraman M, Kopper F, Volkmann J and Deuschl G 2009 Cortical correlates of the basic and first harmonic frequency of Parkinsonian tremor Clin. Neurophysiol. 120 1866-72

[18] Muthuraman M, Heute U, Arning K, Anwar A R, Elble R, Deuschl G and Raethjen J 2012 Oscillating central motor networks in pathological tremors and voluntary movements. What makes the difference? Neuroimage 60 1331-9

[19] Holobar A, Glaser V, Gallego J A, Dideriksen J L and Farina D 2012 Non-invasive characterization of motor unit behaviour in pathological tremor J. Neural Eng. 9056011

[20] Dideriksen J L, Negro F, Enoka R M and Farina D 2012 Motor unit recruitment strategies and muscle properties determine the influence of synaptic noise on force steadiness J. Neurophysiol. $1073357-69$

[21] Christakos C N, Erimaki S, Anagnostou E and Anastasopoulos D 2009 Tremor-related motor unit firing in parkinson's disease: implications for tremor genesis J. Physiol. 587 4811-27

[22] Elek J M, Dengler R, Konstanzer A, Hesse S and Wolf W 1991 Mechanical implications of paired motor unit discharges in pathological and voluntary tremor Electroencephalogr. Clin. Neurophysiol. 81 279-83

[23] Van Cutsem M, Duchateau J and Hainaut K 1998 Changes in single motor unit behaviour contribute to the increase in contraction speed after dynamic training in humans J. Physiol. 513 295-305 
[24] Kudina L and Andreeva R 2013 Motoneuron double discharges: only one or two different entities? Front. Cell Neurosci. 775

[25] Hughes A J, Daniel S E, Kilford L and Lees A J 1992 Accuracy of clinical diagnosis of idiopathic Parkinson's disease: a clinico-pathological study of 100 cases J. Neurol. Neurosurg. Psychiatry 55 181-4

[26] Fahn S and Tolosa E C M 1993 Clinical rating scale for tremor Parkinson's Disease and Movement Disorders 2nd edn, ed J Jankovic and E Tolosa (Baltimore: Williams and Wilkins) pp 271-80

[27] Goetz C C 2003 The unified parkinson's disease rating scale (UPDRS): status and recommendations Mov. Disord. 18 738-50

[28] De Lima E R, Andrade A O, Pons J L, Kyberd P and Nasuto S J 2006 Empirical mode decomposition: a novel technique for the study of tremor time series Med. Biol. Eng. Comput. 44 569-82

[29] Gallego J A, Rocon E, Roa J O, Moreno J C and Pons J L 2010 Real-time estimation of pathological tremor parameters from gyroscope data Sensors 10 2129-39

[30] Holobar A and Zazula D 2007 Multichannel blind source separation using convolution Kernel compensation IEEE Trans. Signal Process. 55 4487-96

[31] Holobar A, Farina D, Gazzoni M, Merletti R and Zazula D 2009 Estimating motor unit discharge patterns from highdensity surface electromyogram Clin. Neurophysiol. 120 551-62

[32] Gallego J A, Dideriksen J L, Holobar A, Ibanez J, Glaser V, Romero J P, Benito-Leon J, Pons J L, Rocon E and Farina D 2015 The phase difference between neural drives to antagonist muscles in essential tremor is associated to the relative strength of supraspinal and afferent input J. Neurosci in press

[33] Holobar A, Minetto M A and Farina D 2014 Accurate identification of motor unit discharge patterns from high-density surface EMG and validation with a novel signal-based performance metric J. Neural Eng. 11 016008

[34] Negro F, Yavuz U Ș and Farina D 2014 Limitations of the spike-triggered averaging for estimating motor unit twitch force: a theoretical analysis PLoS One 9 e92390

[35] Milner-Brown H S, Stein R B and Yemm R 1973 The contractile properties of human motor units during voluntary isometric contractions $J$. Physiol. 228 285-306

[36] Prochazka A, Elek J and Javidan M 1992 Attenuation of pathological tremors by functional electrical stimulation: I. Method Ann. Biomed. Eng. 20 205-24

[37] Gallego J, Dideriksen J, Holobar A, Ibanez J, Pons J, Louis E, Rocon E and Farina D 2014 Influence of common synaptic input to motor neurons on the neural drive to muscle in essential tremor $J$. Neurophysiol. $113182-91$

[38] Farina D, Negro F and Dideriksen J L 2014 The effective neural drive to muscles is the common synaptic input to motor neurons J. Physiol. 49 1-37

[39] Baldissera F, Cavallari P and Cerri G 1998 Motoneuronal precompensation for the low-pass filter characteristics of muscle. A quantitative appraisal in cat muscle units J. Physiol. 511 611-27

[40] Elble R J, Higgins C and Moody C J 1987 Stretch reflex oscillations and essential tremor $J$. Neurol. Neurosurg. Psychiatry 50 691-8

[41] Héroux M E, Pari G and Norman K E 2009 The effect of inertial loading on wrist postural tremor in essential tremor Clin. Neurophysiol. $1201020-9$

[42] Zhang D, Poignet P, Bo A P and Ang W T 2009 Exploring peripheral mechanism of tremor on neuromusculoskeletal model: a general simulation study IEEE Trans. Biomed. Eng. 56 2359-69

[43] Mileusnic M P, Brown I E, Lan N and Loeb G E 2006 Mathematical models of proprioceptors: I. Control and transduction in the muscle spindle J. Neurophysiol. 96 $1772-88$

[44] Hagbarth K E and Young R R 1979 Participation of the stretch reflex in human physiological tremor Brain A J. Neurol. 102 $509-26$

[45] Farina D and Negro F 2011 Accessing the neural drive to muscle and translation to neurorehabilitation technologies IEEE Rev. Biomed. Eng. 5 1-14

[46] Esteki A and Mansour J M 1996 An experimentally based nonlinear viscoelastic model of joint passive moment J. Biomech. $29443-50$

[47] Narici M V, Bordini M and Cerretelli P 1991 Effect of aging on human adductor pollicis muscle function J. Appl. Physiol. (Bethesda, Md. 1985) 71 1277-81

[48] Dideriksen J L, Enoka R M and Farina D 2011 A model of the surface electromyogram in pathological tremor IEEE Trans. Biomed. Eng. $\mathbf{5 8} 2178-85$ 